\title{
Adaptive learning in weighted network games
}

Citation for published version (APA):

Bayer, P., Herings, P. J-J., Peeters, R., \& Thuijsman, F. (2019). Adaptive learning in weighted network games. Journal of Economic Dynamics \& Control, 105, 250-264. https://doi.org/10.1016/j.jedc.2019.06.004

Document status and date:

Published: 01/08/2019

DOI:

10.1016/j.jedc.2019.06.004

Document Version:

Publisher's PDF, also known as Version of record

Document license:

Taverne

Please check the document version of this publication:

- A submitted manuscript is the version of the article upon submission and before peer-review. There can be important differences between the submitted version and the official published version of record.

People interested in the research are advised to contact the author for the final version of the publication, or visit the DOI to the publisher's website.

- The final author version and the galley proof are versions of the publication after peer review.

- The final published version features the final layout of the paper including the volume, issue and page numbers.

Link to publication

\footnotetext{
General rights rights.

- You may freely distribute the URL identifying the publication in the public portal. please follow below link for the End User Agreement:

www.umlib.nl/taverne-license

Take down policy

If you believe that this document breaches copyright please contact us at:

repository@maastrichtuniversity.nl

providing details and we will investigate your claim.
}

Copyright and moral rights for the publications made accessible in the public portal are retained by the authors and/or other copyright owners and it is a condition of accessing publications that users recognise and abide by the legal requirements associated with these

- Users may download and print one copy of any publication from the public portal for the purpose of private study or research.

- You may not further distribute the material or use it for any profit-making activity or commercial gain

If the publication is distributed under the terms of Article $25 \mathrm{fa}$ of the Dutch Copyright Act, indicated by the "Taverne" license above, 


\title{
Adaptive learning in weighted network games
}

\author{
Péter Bayer ${ }^{\mathrm{a}}$, P. Jean-Jacques Herings, ${ }^{\mathrm{b}, *}$, Ronald Peeters ${ }^{\mathrm{c}}$, Frank Thuijsman ${ }^{\mathrm{d}}$ \\ a Laboratoire d'Économie Appliquée de Grenoble, Université Grenoble Alpes, Grenoble Cedex 9 38040, France \\ ${ }^{\mathrm{b}}$ Department of Economics, Maastricht University, P.O. Box 616, Maastricht MD 6200, The Netherlands \\ ${ }^{\mathrm{c}}$ Department of Economics, University of Otago, P.O. Box 56, Dunedin 9054, New Zealand \\ d Department of Data Science and Knowledge Engineering, Maastricht University, P.O. Box 616, Maastricht MD 6200, The Netherlands
}

\section{A R T I C L E I N F O}

\section{Article history:}

Received 20 September 2018

Revised 24 April 2019

Accepted 23 June 2019

Available online 25 June 2019

\section{JEL classification:}

$\mathrm{C} 72$

D74

D83

D85

H41

Keywords:

Networks

Learning

Public goods

Potential games

\begin{abstract}
A B S T R A C T
This paper studies adaptive learning in the class of weighted network games. This class of games includes applications like research and development within interlinked firms, crime within social networks, the economics of pollution, and defense expenditures within allied nations. We show that for every weighted network game, the set of pure Nash equilibria is non-empty and, generically, finite. Pairs of players are shown to have jointly profitable deviations from interior Nash equilibria. If all interaction weights are either non-negative or non-positive, then Nash equilibria are Pareto inefficient. We show that quite general learning processes converge to a Nash equilibrium of a weighted network game if every player updates with some regularity.
\end{abstract}

(C) 2019 Elsevier B.V. All rights reserved.

\section{Introduction}

The theory of learning is of fundamental importance in game theory. With most of the focus in the non-cooperative game theory literature being devoted to the study of equilibria - various concepts, characterizations of the equilibrium set, properties, refinements - it is critical to understand how equilibrium is reached. However, the main concepts of equilibrium theory, and in particular, the concept of Nash equilibrium, have proven difficult to validate, especially in one-shot games. To quote (Fudenberg and Levine, 1998): "One traditional explanation of equilibrium is that it results from analysis and introspection by the players in a situation where the rules of the game, the rationality of the players, and the players' payoff functions are common knowledge. Both conceptually and empirically, these theories have many problems." One of the main goals of learning in game theory is to provide such a motivation. For one-shot games this is typically achieved by interpreting the equilibrium points as results of a series of updates by the players acting in a recurrent setting of that game. These updates are made in response to observed moves by their opponents, with various assumptions on rationality.

\footnotetext{
古 This project is financed by the Centre for Human Enhancement and Learning. We thank Sebastian Bervoets, Yann Bramoullé, Matthieu Faure, and Mark Voorneveld for their extremely useful comments.

* Corresponding author.

E-mail addresses: peter.bayer@univ-grenoble-alpes.fr (P. Bayer), p.herings@maastrichtuniversity.nl (P.J.-J. Herings), ronald.peeters@otago.ac.nz (R. Peeters), f.thuijsman@maastrichtuniversity.nl (F. Thuijsman).
} 
Ideally, as the players discover more about the game and about their opponents, their collective decisions should, in time, resemble equilibrium play. As such, the learning literature focuses mainly on the stability and convergence properties of various learning processes.

The class of games in which we frame our analysis is the class of weighted network games. This class of games generalizes the private provision of local public good games introduced by Bramoullé and Kranton (2007) for undirected graphs and generalized by Bramoullé et al. (2014) for networks with heterogeneous cross-player payoff impacts. For a comprehensive overview of related models, see Sections 3 and 4 of Jackson and Zenou (2014). The main practical reason this class of games is worth studying, is its wide range of applications in various subfields of economic theory, including R\&D within interlinked firms (König et al., 2014), crime within a social network (Ballester et al., 2006), and peer effects with spatial interactions (Blume et al., 2010). Further applications include defense expenditures within an international community as studied by Sandler and Hartley (1995, 2007).

Networks offer a simple way to model complex interactions between many decision makers. The simplest network models are undirected graphs, in which a link between a pair of players indicates a direct interaction. Since players may be indirectly affected by the neighbors of their neighbors, and so on, each interaction may be relevant for each player, resulting in a profoundly rich model. In weighted networks, interaction weights with arbitrary values, either positive or negative, are used to characterize the way that pairs of interacting players influence each other.

The parameters of a weighted network game are the weighted network itself, describing the interactions between the players, a vector of targets that describes the players' needs, and a vector of upper bounds representing the players' highest possible activity levels. Each player has a concave benefit function of the weighted aggregate activity and a linear cost function of his own activity. We show that the set of Nash equilibria of weighted network games is non-empty and generically finite. Additionally, we show that under quite general conditions pairs of players can jointly improve their payoffs, so Nash equilibria are not strong. We also give conditions such that they are not Pareto efficient.

We study a class of learning processes with the following features. The players update their decisions at discrete points in time, maximizing their payoffs for a single period. The updates determine the status quo of the next period. At any given period, only one player is allowed to update, whereas the actions of every other player remain the same. This class of learning processes includes e.g. the improvement paths of Monderer and Shapley (1996).

Weighted network games are generalized aggregative games (Dubey et al., 2006) as well as best-response potential games (Voorneveld, 2000), but may not belong to the class of ordinal potential games (Monderer and Shapley, 1996). Since weighted network games generally do not have an ordinal potential, better-response dynamics may not converge, and we show the possibility of non-convergence by an example.

Our main results concern the properties of adaptive learning processes centered around the best responses. We find that convergence to the set of Nash equilibria requires two conditions: (1) each update has to take the player closer to his contemporary best response, and (2) with some regularity, every player must have the possibility to update. Furthermore, we show that such processes converge to a Nash equilibrium point if (3) the set of Nash equilibria is finite. The first and second conditions concern the players and may be interpreted as assumptions of cautiousness and activity, respectively. The third condition concerns the parameters of the weighted network game and is generically satisfied. The main significance of our results is in the fact that the convergence of learning processes to a Nash equilibrium can be achieved with relatively weak assumptions on the behavior of the players. Our convergence conditions are less demanding than those studied before and weakening either of (1) or (2) would lead to non-convergence. Furthermore, our results are achieved for a wider class of games than those appearing in the existing literature. Crucially, generic finiteness of the Nash equilibrium set - and therefore, our strongest convergence theorem - is shown for all networks, covering previously explored and unexplored but economically relevant classes of games.

Our class of learning processes with cautious improvements resembles Selten's directional learning model (Selten and Buchta, 1998; Selten and Stoecker, 1986) which is often illustrated by the archer trying to hit the trunk of a tree aiming more to the left (right) with the next arrow if the previous arrow passed the trunk to the right (left). Various studies have successfully applied this qualitative learning theory to explain learning behavior in experimental data; see Cachon and Camerer (1996) for coordination games, Cason and Friedman (1997) for markets, Kagel and Levin (1999) for auctions, and Nagel and Vriend (1999) for oligopoly markets.

As revision opportunities are generally scarce in real-life applications, the analysis of general, discrete-time learning processes are necessary to provide motivation for the use of Nash equilibria. To our knowledge, our paper is the first to do so in the setting of weighted network games. Bramoullé et al. (2014) and Bervoets and Faure (2019) study best-response dynamics in continuous time in the restricted class of weighted network games with all weights being equal to 0 or to $\delta \in(0,1)$. Bervoets et al. (2018) considers a two-stage stochastic learning process with experimenting players that converges with probability one. Eksin et al. (2012) considers a similar game of incomplete information played on a graph. Unlike in previous papers, where players' updates happened continuously, our players may make erratic jumps, such as overshooting their best responses. On a technical level, this means that our results are achieved by exploiting the global properties of the best-response potential, rather than the local ordinal property as used by Bervoets and Faure (2019). The erratic jumps may represent mistakes made by the players either through a "slip of the hand" or through their inability to calculate optimal strategies due to a lack of sophistication. Therefore, both our setting and our results are behaviorally motivated. 
The paper proceeds as follows. Section 2 introduces weighted network games. Section 3 contains the characterization of the set of Nash equilibria and its welfare properties. In Section 4 we define learning processes and discuss their cycling properties. Section 5 contains our main results, the convergence conditions of learning processes. Section 6 concludes.

\section{Weighted network games}

Let $I=\{1, \ldots, n\}$ denote the set of players with $n \geq 2$. The action set of player $i \in I$ is $X_{i}=\left[0, \bar{x}_{i}\right]$ with $\bar{x}_{i}>0$. Let $x_{i} \in X_{i}$ denote player $i$ 's action. The action profile of all players is denoted by $x=\left(x_{j}\right)_{j \in I}$ and the action profile of all players except $i$ by $x_{-i}=\left(x_{j}\right)_{j \neq i}$. Similarly, $X=\prod_{i \in I} X_{i}$ denotes the set of action profiles and $X_{-i}=\prod_{j \in I \backslash\{i\}} X_{j}$ the set of action profiles for all players other than $i$.

Definition 2.1. The tuple $G=\left(I, X,\left(\pi_{i}\right)_{i \in I}\right)$ is called a weighted network game if for every $i \in I$ the payoff function $\pi_{i}: X \rightarrow \mathbb{R}$ is given by:

$$
\pi_{i}(x)=f_{i}\left(\sum_{j \in I} w_{i j} x_{j}\right)-c_{i} x_{i},
$$

with cost parameters $c_{i}>0$, interaction weights $w_{i j} \in \mathbb{R}$, and benefit functions $f_{i}: \mathbb{R} \rightarrow \mathbb{R}$.

Assumption 2.2. For every $i \in I, w_{i i}=1$, and for every $i, j \in I, w_{i j}=w_{j i}$. Furthermore, for every $i \in I$, the benefit function $f_{i}$ is twice continuously differentiable and satisfies the following properties: (1) $f_{i}^{\prime}>0$, (2) $f_{i}^{\prime \prime}<0$, and (3) there exists $t_{i} \in \mathbb{R}$ such that $f_{i}^{\prime}\left(t_{i}\right)-c_{i}=0$.

The interpretation is the following. Each player $i \in I$ produces a specialized good using a linear production technology. The costs of producing one unit of the good are equal to $c_{i}$. The production of player $i$ is denoted by $x_{i}$. Each player consumes his own good as well as a weighted sum of his neighbors' goods. The total amount of consumption of player $i$ is $\sum_{j \in I} w_{i j} x_{j}$ and the benefit of consumption is $f_{i}\left(\sum_{j \in I} w_{i j} x_{j}\right)$. The consumption level $t_{i}$ where the marginal benefits of consumption are equal to $c_{i}$ is called the target value. Note that since $t_{i}$ can be above or below values achievable by using action profiles in $X$, property (3) of the benefit function is without loss of generality.

For player $i$, the interaction weight $w_{i j}$ captures the substitutability of one unit of player $j$ 's good to his own. If $w_{i j}>0$, then player $j$ 's production generates positive externalities for player $i$. Specifically, if $w_{i j}=1$, then player $i$ 's enjoyment of player $j$ 's good equals that of his own good. If $w_{i j} \in(0,1)$, then player $i$ derives less benefits from player $j$ 's good than from his own. If $w_{i j} \in(1, \infty)$, then player $i$ enjoys the good of player $j$ more than his own. Negative values of $w_{i j}$ indicate that player $j$ 's production has negative external effects on player $i$ 's benefits, with $w_{i j} \in(0,-1), w_{i j}=-1$, and $w_{i j} \in(-1,-\infty)$ indicating that the negative effects are smaller, equal, or greater in magnitude than the positive effects of equal amounts of the own good. The assumption $w_{i i}=1$ is a normalization. The symmetry assumption $w_{i j}=w_{j i}$ for $i, j \in I$ is also made in previous studies like Dubey et al. (2006), Bramoullé and Kranton (2007), and Bramoullé et al. (2014). The asymmetric case $w_{i j} \neq w_{j i}$ is relatively unexplored in the local public good setting and hence an interesting direction for future research, see Bourlès et al. (2017) for a model of transfers with asymmetric interactions. The interaction weights are collected in a matrix W.

Example 2.3. Let $I=\{1,2\}$ be a set of two countries that have to decide on the level of their defense expenditures. We take $X_{i}=\left[0, Z_{i}\right]$, where $Z_{i}$ denotes the GDP of Country $i \in I$, and

$$
W=\left(\begin{array}{cc}
1 & w_{12} \\
W_{12} & 1
\end{array}\right)
$$

The increasing, concave functions $f_{1}$ and $f_{2}$ indicate the countries' benefits from defense. Let $t_{i}=0.01 Z_{i}$, indicating that both countries have a target value for defense expenditures of $1 \%$ of their GDP. This is the amount they would spend on defense if the other nation spends nothing.

If $w_{12}=0$, then neither country benefits from the other's defense expenditures, nor are they threatened by it. This may indicate neutrality or a significant geographical distance. If $w_{12}>0$, the two nations are allies and the game becomes one of strategic substitutes. In this case both nations benefit from the other's defense spending and national defense expenditures are likely to be lower than $1 \%$ of GDP. If $w_{12}<0$, the two nations are hostile to each other, and the game is one of strategic complements. In this case the nations are hurt or threatened by the other's defense spending and defense expenditures will likely exceed $1 \%$ of GDP.

In case $w_{12}=1$, Example 2.3 results in the 2-player pure public good model of defense expenditures between allies, while $0<w_{12}<1$ gives the symmetric version of the limited substitutability public good model of defense expenditures between allies, developed by Sandler and Hartley (2001). They do not consider the case $w_{12}<0$. In the subsequent section, we discuss how the set of Nash equilibria of this particular game depends on the parameters $t_{1}, t_{2}$, and $w_{12}$ in more detail.

Our set-up allows the modeling of more intricate relationships between players, as illustrated by the following example. 
Example 2.4. Let $I=\{1,2,3\}$ be a set of three countries deciding on the level of their defense expenditures, $X_{i}=\left[0, Z_{i}\right]$, and

$$
W=\left(\begin{array}{ccc}
1 & -1 & 1 \\
-1 & 1 & 1 \\
1 & 1 & 1
\end{array}\right)
$$

and $t=\left(0.03 Z_{1}, 0.03 Z_{2}, 0.01 Z_{3}\right)$. In this example, Countries 1 and 2 are rivals, but both of them are friendly to Country 3. An example of this type of relationship may be that of Israel, Saudi Arabia, and the U.S. since the 2000s.

Example 2.4 and similar examples that feature intransitive relationships between countries cannot be modeled in the spirit of Sandler and Hartley (2001). Our set-up therefore reflects more closely the possible intricacies of diplomatic relationships and can be used to model any system of alliances and threats, provided that the relationship between any two nations is symmetric.

Games with strategic substitutes and complements are of great relevance in the economic literature. The game of Example 2.3 can be interpreted as a game where two firms choose their output to maximize their profits, with the interaction weight $w_{12}$ deciding whether their products are net substitutes or net complements. An interaction matrix similar to the one of Example 2.4 may describe the relationship between two competitor firms producing substitute goods, e.g. plane manufacturers Airbus and Boeing, and a third firm producing a complementary good, e.g. a kerosene supplier Exxon Mobil. Other such examples include gaming consoles, XBox and Playstation with a game developer EA Sports, or tea companies Lipton and Twinings with a sugar company Südzucker. Weighted network games provide a framework to model any type of relationship structure with any number of companies. As it will be apparent later, our main results extend to Bertrand and Cournot games of differentiated products and linear demand curves, since weighted network games are best-response equivalent to such games.

We denote the set of weighted network games satisfying Assumption 2.2 by $\mathcal{G}$. Since $w_{i i}=1$ for every $i \in I$ and $w_{i j}=$ $w_{j i}$ for every $i, j \in I$, the number of free parameters in $W$ is $n(n-1) / 2$. Let $w=\left(w_{i j}\right)_{i, j \in I, i<j} \in \mathbb{R}^{n(n-1) / 2}$ denote the upper triangular elements of $W$. We define the set of parameters $P=\mathbb{R}_{++}^{n} \times \mathbb{R}^{n(n-1) / 2} \times \mathbb{R}^{n}$. Then, for $(\bar{x}, w, t) \in P$, let $\mathcal{G}(\bar{x}, w, t)$ be the set of weighted network games in $\mathcal{G}$ with upper bounds $\bar{x}$, interaction weights $w$, and targets $t$. A weighted network game in such a set is characterized by the benefit functions $f_{i}$ and cost parameters $c_{i}$.

The properties in Assumption 2.2 imply that for every $x_{-i} \in X_{-i}, \pi_{i}\left(x_{i}, x_{-i}\right)$ has a unique global maximizer in $X_{i}$. For player $i \in I$, let $b_{i}: X \rightarrow X_{i}$ denote his best-response function, i.e. $b_{i}(x)=\operatorname{argmax}_{x_{i} \in X_{i}} \pi_{i}\left(x_{i}, x_{-i}\right)$ for every $x \in X$. We now show that for a fixed configuration $(\bar{x}, w, t) \in P$, all games in $\mathcal{G}(\bar{x}, w, t)$ are best-response equivalent, which implies that all games in $\mathcal{G}(\bar{x}, w, t)$ have the same set of Nash equilibria.

Proposition 2.5. Let $(\bar{x}, w, t) \in P$ and let $G \in \mathcal{G}(\bar{x}, w, t)$ be a weighted network game. For every $i \in I$ and $x \in X$ it holds that

$$
b_{i}(x)= \begin{cases}0 & \text { if } t_{i}-\sum_{j \neq i} w_{i j} x_{j}<0, \\ t_{i}-\sum_{j \neq i} w_{i j} x_{j} & \text { if } t_{i}-\sum_{j \neq i} w_{i j} x_{j} \in\left[0, \bar{x}_{i}\right], \\ \bar{x}_{i} & \text { if } t_{i}-\sum_{j \neq i} w_{i j} x_{j}>\bar{x}_{i} .\end{cases}
$$

It is useful to define a player's unconstrained best response, the contribution level a player would choose if instead of $\left[0, \bar{x}_{i}\right]$, the set of available actions were equal to $\mathbb{R}$. For player $i \in I$ and action profile $x \in X$, let $\widehat{b}_{i}(x)=t_{i}-\sum_{j \neq i} w_{i j} x_{j}$ denote this value. The unconstrained best response of a player $i$ is such that his contribution $\widehat{b}_{i}(x)$ together with the weighted contributions of the other players $\sum_{j \neq i} w_{i j} x_{j}$ is equal to his target value $t_{i}$. Clearly, $\widehat{b}_{i}(x) \neq b_{i}(x)$ implies that $t_{i}-\sum_{j \neq i} w_{i j} x_{j} \notin$ $\left[0, \bar{x}_{i}\right]$ and therefore the actual best response is on the boundary: $b_{i}(x) \in\left\{0, \bar{x}_{i}\right\}$.

For $i \in I$, we define the numbers $\underline{b}_{i}$ and $\bar{b}_{i}$ by $\underline{b}_{i}=\min _{x_{-i} \in X_{-i}}\left(t_{i}-\sum_{j \neq i} w_{i j} x_{j}\right)$ and $\bar{b}_{i}=\max _{x_{-i} \in X_{-i}}\left(t_{i}-\sum_{j \neq i} w_{i j} x_{j}\right)$. Since the set $X_{-i}$ is compact, both $\underline{b}_{i}$ and $\bar{b}_{i}$ are well-defined. It is easily seen that the unconstrained best response of player $i$ always belongs to the interval $\left[\underline{b}_{i}, \bar{b}_{i}\right]$.

Proposition 2.5 shows that for every player $i \in I$ and every action profile $x \in X$ such that $b_{i}(x) \in\left(0, \bar{x}_{i}\right)$, ceteris paribus changing player $j$ 's action by $\Delta x_{j}$ changes player $i$ 's best response by $-w_{i j} \Delta x_{j}$.

\section{Nash equilibria}

Since the paper's main focus is on the convergence of adaptive learning processes to the set of Nash equilibria, as a precursor we characterize the relevant properties of this set.

We first show that a weighted network game $G \in \mathcal{G}$ is a best-response potential game (Voorneveld, 2000). A game with set of players $I$, action space $X$, and payoff functions $\left(\pi_{i}\right)_{i \in I}$ is a best-response potential game if there exists a function $\phi: X \rightarrow \mathbb{R}$ such that for every $i \in I$ and every $x \in X$ it holds that

$$
\underset{x_{i} \in X_{i}}{\operatorname{argmax}} \pi_{i}\left(x_{i}, x_{-i}\right)=\underset{x_{i} \in X_{i}}{\operatorname{argmax}} \phi\left(x_{i}, x_{-i}\right) .
$$


We call $\phi$ the best-response potential of game $(I, X, \pi)$. In the following proposition we show that every weighted network game is a best-response potential game and use $\phi$ to characterize the set of Nash equilibria. For $(\bar{x}, w, t) \in P$, let $\operatorname{NE}(\bar{x}, w, t)$ denote the set of Nash equilibria of a game in $\mathcal{G}(\bar{x}, w, t)$.

Proposition 3.1. For every $(\bar{x}, w, t) \in P$, it holds that:

1. Every game $G \in \mathcal{G}(\bar{x}, w, t)$ is a best-response potential game with potential $\phi: X \rightarrow \mathbb{R}$ defined by

$$
\phi(x)=x^{\top} t-\frac{1}{2} x^{\top} W x, \quad x \in X .
$$

2. $\mathrm{NE}(\bar{x}, w, t) \neq \emptyset$.

3. $x^{*} \in \mathrm{NE}(\bar{x}, w, t)$ if and only if $x^{*}$ satisfies the Karush-Kuhn-Tucker conditions for the optimization problem $\max _{x \in X} \phi(x)$, i.e. for every $i \in I$, there exist $\lambda_{i}, \mu_{i} \in \mathbb{R}_{+}$such that

$$
\begin{aligned}
& t_{i}-\sum_{j \in I} w_{i j} x_{j}^{*}+\lambda_{i}-\mu_{i}=0, \\
& x_{i}^{*} \geq 0, \quad \bar{x}_{i} \geq x_{i}^{*}, \\
& \lambda_{i} x_{i}^{*}=0, \quad \mu_{i}\left(\bar{x}_{i}-x_{i}^{*}\right)=0 .
\end{aligned}
$$

The best-response potential allows for a simple characterization of the set of Nash equilibria as the solution set of a KKT problem. The existence of a Nash equilibrium immediately follows due to the bounded action space. In case the action space is unbounded, negative interaction weights may cause an infinite increase of best replies, leading to nonexistence.

The first part of Proposition 3.1 is related to Lemma 1 of Bramoullé et al. (2014) which applies to weighted network games where all weights equal 0 or $\delta \in(0,1)$ and for which $\phi$ is an ordinal potential in the sense of Monderer and Shapley (1996). Weighted network games in our class $\mathcal{G}$ do not generally admit an ordinal potential. In fact, Example 4.3 shows that better-response dynamics can lead to cycles in our set-up, which is incompatible with the existence of an ordinal potential. Proposition 3.1 therefore only makes the weaker claim that $\phi$ is a best-response potential.

The existence of a best-response potential is of particular interest for a wide class of games. Their significance in the aggregative/network games strand of literature was first pointed out by Kukushkin (2004). Ewerhart (2017) studies this question in the class of contest games. Since weighted network games are also generalized aggregative games (Jensen, 2010), the existence of the best-response potential also follows from more general results in the literature. In particular, it can be shown that the pseudo-potential of Theorem 1 in Dubey et al. (2006) simplifies into the quadratic function $\phi$.

In addition to $\phi$ being a best-response potential, for network games on unweighted graphs, Bervoets and Faure (2019) show that locally, $\phi$ serves as an ordinal potential, i.e:

$$
\operatorname{sgn}\left(\frac{\partial \pi_{i}\left(x_{i}, x_{-i}\right)}{\partial x_{i}}\right)=\operatorname{sgn}\left(\frac{\partial \phi\left(x_{i}, x_{-i}\right)}{\partial x_{i}}\right) .
$$

This property can be easily generalized for weighted network games.

Using the characterization in the third part of Proposition 3.1, we show that for any vector of bounds $\bar{x}$ and any interaction structure $w$, the set of target vectors $t$ that induce infinitely many Nash equilibria is of null measure.

Proposition 3.2. For every $\bar{x} \in \mathbb{R}_{++}^{n}$, for every $w \in \mathbb{R}^{n(n-1) / 2}$, for almost every $t \in \mathbb{R}^{n}$, the weighted network game $G \in \mathcal{G}(\bar{x}, w, t)$ has a finite number of Nash equilibria.

In the appendix we show that the set of target vectors with infinitely many Nash equilibria is not only small in a measure theoretic sense, but also in a topological sense.

The generic finiteness of the set of Nash equilibria is illustrated in the following example. For $H \subseteq I, \operatorname{let}^{\mathrm{NE}_{H}}(\bar{x}, w, t)$ be the set of Nash equilibria associated with Lagrange parameters $\lambda_{i}, \mu_{i}$ in Proposition 3.1 such that for all $i \in H, \lambda_{i}=\mu_{i}=0$ and for all $i \in I \backslash H, \max \left\{\lambda_{i}, \mu_{i}\right\}>0$. In words, $\mathrm{NE}_{H}(\bar{x}, w, t)$ denotes the set of Nash equilibria such that players outside $H$ are either contributing zero or contributing up their capacity and players inside $H$ are choosing an interior contribution level.

Example 3.3. Fix parameters $\bar{x}$ and $w_{12}$ in a weighted network game with two players. We have shown in Proposition 3.2 that for almost every $t \in \mathbb{R}^{2}$ the set $\mathrm{NE}\left(\bar{x}, w_{12}, t\right)$ is finite. For every $t \in \mathbb{R}^{2}$, the set $\mathrm{NE}_{\emptyset}\left(\bar{x}, w_{12}, t\right)$ is trivially finite, and it is easy to see that the sets $\mathrm{NE}_{\{1\}}\left(\bar{x}, w_{12}, t\right)$ and $\mathrm{NE}_{\{2\}}\left(\bar{x}, w_{12}, t\right)$ are finite in the case of two players.

We therefore only check the interior solutions to the KKT problem of this game as defined in Proposition 3.1, i.e. where all Lagrange parameters $\lambda_{i}, \mu_{i}$ are zero. In an interior solution $x^{*} \in \mathrm{NE}_{\{1,2\}}\left(\bar{x}, w_{12}, t\right)$, we have $\widehat{b}_{1}\left(x^{*}\right)=x_{1}^{*}$ and $\widehat{b}_{2}\left(x^{*}\right)=x_{2}^{*}$, therefore

$$
\begin{aligned}
& x_{1}^{*}=t_{1}-w_{12} x_{2}^{*}, \\
& x_{2}^{*}=t_{2}-w_{12} x_{1}^{*} .
\end{aligned}
$$

In case $w_{12}$ is not equal to 1 or -1 , rearranging yields

$$
\begin{aligned}
& x_{1}^{*}=\frac{t_{1}-w_{12} t_{2}}{1-\left(w_{12}\right)^{2}}, \\
& x_{2}^{*}=\frac{t_{2}-w_{12} t_{1}}{1-\left(w_{12}\right)^{2}} .
\end{aligned}
$$


Therefore, for every $w_{12} \in \mathbb{R} \backslash\{-1,1\}$, we have $\left|\mathrm{NE}_{\{1,2\}}\left(\bar{x}, w_{12}, t\right)\right| \leq 1$. Whether or not the set of interior equilibria is empty depends on whether $x^{*}$ is an element of $X$.

If $w_{12}=-1$, it is easy to check that $t_{1}=t_{2}=0$ yields infinitely many Nash equilibria $x^{*}$ with $x_{1}^{*}=x_{2}^{*}$. There can also be infinitely many Nash equilibria when $t_{1}+t_{2}=0$. There are no interior Nash equilibria for different values of $t$ as then the system of best responses is inconsistent. Similarly, if $w_{12}=1$ then there can only be infinitely many Nash equilibria if $t_{1}=t_{2}$. Indeed, consider the case with $t_{1}=t_{2}$. If $\bar{x}_{1}+\bar{x}_{2}>t_{1}$, then there are infinitely many Nash equilibria $x^{*}$ with $x_{1}^{*}+x_{2}^{*}=t_{1}$, if $\bar{x}_{1}+\bar{x}_{2}=t_{1}$, then there is a unique interior Nash equilibrium, and if $\bar{x}_{1}+\bar{x}_{2}<t_{1}$, then there are no interior Nash equilibria.

The proof of our result follows the approach of Example 3.3. We partition the players in two groups, those who choose an interior production level and those who choose a corner solution. For each possible partition, the set of equilibria consistent with that partition is generically finite. The result follows from the fact that there is a finite number of possible partitions. Ballester and Calvó-Armengol (2010), Belhaj et al. (2014) and Allouch (2015) provide results for the uniqueness of Nash equilibrium.

We conclude this section by discussing efficiency properties of equilibria. We consider efficiency in the Pareto sense. Other models consider efficiency in terms of minimizing total efforts/production (Bramoullé and Kranton, 2007; Goyal, 2012) or maximizing total welfare (Bramoullé and Kranton, 2007; Helsley and Zenou, 2014). We first show that a pair of players with a non-zero interaction weight can always jointly deviate from an interior equilibrium to a better action profile. For a subset of players $H \subseteq I$ and $\delta \in \mathbb{R}$, let $\delta^{H} \in \mathbb{R}^{n}$ denote the vector such that $\delta_{i}^{H}=\delta$ for $i \in H$ and $\delta_{i}^{H}=0$ for $i \in I \backslash H$.

Proposition 3.4. Let $(\bar{x}, w, t) \in P$ be given. Let $x^{*} \in \mathrm{NE}(\bar{x}, w, t)$ be a Nash equilibrium of a game $G=(I, X, \pi) \in \mathcal{G}(\bar{x}, w, t)$ such that $x_{i}^{*} \in\left(0, \bar{x}_{i}\right)$ and $x_{j}^{*} \in\left(0, \bar{x}_{j}\right)$. It holds that:

1. If for some $i, j \in I$ with $i \neq j$ it holds that $w_{i j} \neq 0$, then there exists $\delta \in\left(0, \min \left\{x_{i}^{*}, x_{j}^{*}, \bar{x}_{i}-x_{i}^{*}, \bar{x}_{j}-x_{j}^{*}\right\}\right)$ such that $\pi_{i}\left(x^{*}\right)<$ $\pi_{i}\left(x^{*}+\operatorname{sgn}\left(w_{i j}\right) \delta^{\{i, j\}}\right)$ and $\pi_{j}\left(x^{*}\right)<\pi_{j}\left(x^{*}+\operatorname{sgn}\left(w_{i j}\right) \delta^{\{i, j\}}\right)$.

2. If $w \geq 0$ or $w \leq 0$ and, for some $i, j \in I$ with $i \neq j$, it holds that $w_{i j} \neq 0$, then there exists $\delta \in\left(0, \min \left\{x_{i}^{*}, x_{j}^{*}, \bar{x}_{i}-x_{i}^{*}, \bar{x}_{j}-x_{j}^{*}\right\}\right)$ such that the action profile $x^{*}+\operatorname{sgn}\left(w_{i j}\right) \delta^{\{i, j\}}$ is a Pareto improvement over $x^{*}$.

Proposition 3.4 implies that interior Nash equilibria are not strong Nash equilibria since there are profitable deviations by coalitions of two linked players. Moreover, interior Nash equilibria are not Pareto efficient provided that the interaction weights are either all non-negative or all non-positive. See Elliott and Golub (2019) for a characterization of efficient Nash equilibria in the non-negative case.

\section{Cycling of learning processes}

Within the framework of weighted network games, we consider learning processes where players update their strategies sequentially. That is, given some initial action profile, one player changes his action, while that of every other player remains the same. Then, another player makes a change under similar circumstances, and so on. We improve upon existing results (Dubey et al., 2006; Kukushkin, 2004), which show acyclicity of best-response dynamics by identifying the conditions under which a general class of learning processes (particularly, the better-response dynamics) may cycle. We find that learning processes may cycle if and only if players move away from or radically overshoot the best response. Since the non-existence of best-response cycles is a necessary but not sufficient condition of the convergence of best-response dynamics (Kukushkin, 2015), this is a crucial step towards our convergence results.

Let $\mathbb{N}=\{1,2, \ldots\}$ denote the set of positive integers and let $\mathcal{K}=\{\{1,2\},\{1,2,3\}, \ldots\} \cup \mathbb{N}$ be a collection of index sets. For $K \in \mathcal{K}$, if $K=\mathbb{N}$, then we define $K^{-}=K$, and if there is $m \in \mathbb{N}$ such that $K=\{1, \ldots, m\}$, then define $K^{-}=K \backslash\{m\}$ as the set that results from $K$ by leaving out its highest element.

Definition 4.1. Let some $G \in \mathcal{G}$ and $K \in \mathcal{K}$ be given. A sequence of action profiles $\left(x^{k}\right)_{k \in K}$ is a path in the game $G$ if:

1. For each $k \in K^{-}$there exists a player $i^{k}$ such that $x_{-i^{k}}^{k+1}=x_{-i^{k}}^{k}$.

2. There is at least one $k \in K^{-}$such that $x^{k+1} \neq x^{k}$.

If $x_{-i^{k}}^{k+1}=x_{-i^{k}}^{k}$, and $x_{i^{k}}^{k+1} \neq x_{i^{k}}^{k}$, then we call $i^{k}$ the updating player at period $k$. For $K=\{1, \ldots, m\} \in \mathcal{K}$, a path $\left(x^{k}\right)_{k \in K}$ in the game $G$ is a cycle if $x^{1}=x^{m}$.

As per Definition 4.1, a path is a sequence where at most one player has changed his contribution between any two successive action profiles, while there are at least two different action profiles in the sequence.

Definition 4.2. Let some $G \in \mathcal{G}$ and $K \in \mathcal{K}$ be given. A path $\left(x^{k}\right)_{k \in K}$ is best-response compatible in the game $G$ if for every $k \in K^{-}$it holds that:

1. If $x^{k+1}=x^{k}$, then there exists $i^{k} \in I$ such that $x_{i^{k}}^{k+1}=x_{i^{k}}^{k}=b_{i^{k}}\left(x^{k}\right)$.

2. If $x_{i^{k}}^{k+1} \neq x_{i^{k}}^{k}$, then $x_{i^{k}}^{k+1}=b_{i^{k}}\left(x^{k}\right)$. 
A path $\left(x^{k}\right)_{k \in K}$ is better-response compatible in the game $G$ if for every $k \in K^{-}$it holds that:

1. If $x^{k+1}=x^{k}$, then there exists $i^{k} \in I$ such that $x_{i^{k}}^{k+1}=x_{i^{k}}^{k}=b_{i^{k}}\left(x^{k}\right)$.

2. If $x_{i^{k}}^{k+1} \neq x_{i^{k}}^{k}$, then $\pi_{i^{k}}\left(x^{k+1}\right)>\pi_{i^{k}}\left(x^{k}\right)$.

Definition 4.2 captures two of the simplest and best-known learning processes. In case of a best-response compatible path, each updating player moves to his best available option. In case of a better-response compatible path, updating players are only required to strictly improve their payoffs. Clearly, a best-response compatible path is also a better-response compatible path.

It is well known that best-response dynamics do not produce cycles in best-response potential games (Voorneveld, 2000), which includes weighted network games by Proposition 3.1. Better-response dynamics do not generate cycles in ordinal potential games (Monderer and Shapley, 1996). The following example shows that better-response cycles can occur within weighted network games.

Example 4.3. Let $I=\{1,2\}, X_{1}=X_{2}=[0,4]$, and $t_{1}=t_{2}=1$. Moreover, let the payoff functions be given by

$$
\pi_{1}\left(x_{1}, x_{2}\right)=2 \sqrt{x_{1}+0.6 x_{2}}-x_{1}
$$

and

$$
\pi_{2}\left(x_{1}, x_{2}\right)=2 \sqrt{x_{2}+0.6 x_{1}}-x_{2} \text {. }
$$

It is easy to check that $\pi_{1}$ and $\pi_{2}$ satisfy the properties laid down in Definition 2.1 and Assumption 2.2 with $w_{12}=0.6$, $f_{1}(z)=f_{2}(z)=2 \sqrt{z}$, and $c_{1}=c_{2}=1$, except that the benefit functions are not defined on all of $\mathbb{R}$ and are not differentiable at $z=0$. This is not problematic since all relevant values of $z$ are greater than or equal to 0.06 in what follows.

Table 1 presents a sequence of action profiles that constitutes a better-response cycle for this example.

Note that the changes in player 1's choice of actions between periods 1 and 2 and between periods 3 and 4, as well as those for player 2 between periods 4 and 5 and between periods 6 and 7 are quite large, given the action space. Columns 5 and 6 of Table 2 present for each period the distance between the current action and both the best response and the action chosen by the player updating his action. Notice that in periods 1 and 4, the actions chosen are more than twice as far away from the current action than the best response is, meaning that the updating player, despite the increase in payoffs, has moved farther from his optimal decision than he originally was. We refer to this as extreme overshooting beyond the best response. The paper's main result is showing that a lack of extreme overshooting is a sufficient and necessary condition of convergence in the class of learning processes centered around the best responses.

As before, for a path $\left(x^{k}\right)_{k \in K}$, let $\left(i^{k}\right)_{k \in K^{-}}$denote the updating player in period $k$ if there was a change in the action profile; if there was no change, let $i^{k}$ be a best-responding player if such a player exists and an arbitrarily chosen player otherwise. Furthermore, for $k \in K^{-}$, let the overshooting coefficient $\alpha_{k} \in \mathbb{R} \cup\{-\infty, \infty\}$ be defined as

$$
\alpha_{k}=\frac{x_{i^{k}}^{k+1}-b_{i^{k}}\left(x^{k}\right)}{x_{i^{k}}^{k}-b_{i^{k}}\left(x^{k}\right)} .
$$

Table 1

Actions played and payoffs in the better-response cycle in Example 4.3.

\begin{tabular}{lllll}
\hline$k$ & $x_{1}^{k}$ & $x_{2}^{k}$ & $\pi_{1}\left(x^{k}\right)$ & $\pi_{2}\left(x^{k}\right)$ \\
\hline 1 & 0 & 0.1 & 0.49 & 0.53 \\
2 & 3 & 0.1 & 0.50 & 2.66 \\
3 & 3 & 0 & 0.46 & 2.68 \\
4 & 0.1 & 0 & 0.53 & 0.49 \\
5 & 0.1 & 3 & 2.66 & 0.50 \\
6 & 0 & 3 & 2.68 & 0.46 \\
7 & 0 & 0.1 & 0.49 & 0.53 \\
\hline
\end{tabular}

Table 2

The size of action changes in the better-response cycle of Table 1.

\begin{tabular}{lllllll}
\hline$k$ & $x_{1}^{k}$ & $x_{2}^{k}$ & $b_{i^{k}}\left(x^{k}\right)$ & $\left|b_{i^{k}}\left(x^{k}\right)-x_{i^{k}}^{k}\right|$ & $\left|x_{i^{k}}^{k+1}-x_{i^{k}}^{k}\right|$ & $\alpha_{k}$ \\
\hline 1 & 0 & 0.1 & 0.94 & 0.94 & 3 & -2.19 \\
2 & 3 & 0.1 & 0 & 0.1 & 0.1 & 0 \\
3 & 3 & 0 & 1 & 2 & 2.9 & -0.45 \\
4 & 0.1 & 0 & 0.94 & 0.94 & 3 & -2.19 \\
5 & 0.1 & 3 & 0 & 0.1 & 0.1 & 0 \\
6 & 0 & 3 & 1 & 2 & 2.9 & -0.45 \\
7 & 0 & 0.1 & & & & \\
\hline
\end{tabular}


In case the denominator is 0 , we take the convention that $\alpha_{k}=-\infty$ if the numerator is negative, $\alpha_{k}=0$ if the numerator is 0 , and $\alpha_{k}=+\infty$ if the numerator is positive. Column 7 of Table 2 shows the values of $\alpha_{k}$ in the better-response cycle of Example 4.3.

The coefficient $\alpha_{k}$ determines the extent of overshooting of the updating player beyond the best response. If there is overshooting, then $\alpha_{k}$ is negative. There is no overshooting if $\alpha_{k}$ is positive. If $\alpha_{k}=0$ then the updating player moved to the best response. If $\alpha_{k} \in\{-\infty, \infty\}$ then $x_{i^{k}}^{k+1} \neq x_{i^{k}}^{k}=b_{i^{k}}\left(x^{k}\right)$, so the player moved away from a best response. If $\alpha_{k}<-1$, then the new action is farther from the best response relative to the action before the update.

Values of $\alpha_{k}$ in $(0,1)$ correspond to a better response, while in case $\alpha_{k}>1$ the payoff of the updating player is lower than before. For negative values of $\alpha_{k}$, the threshold between better and worse replies depends on the payoff function. The possible values that $\alpha_{k}$ may take depend on $\bar{x}$.

As suggested by Example 4.3, sequences of action profiles that feature extreme overshooting beyond the best response may cycle. We therefore characterize sequences by their extent of overshooting.

Definition 4.4. A path $\left(x^{k}\right)_{k \in K}$ in a game $G \in \mathcal{G}$ is $\alpha$-centered for some $\alpha>0$ if for every $k \in K^{-}$it holds that $\left|\alpha_{k}\right|<\alpha$.

A best-response compatible path is $\alpha$-centered for every $\alpha>0$. Furthermore, for every $\alpha>0$ there exist paths that are better-response compatible and $\alpha$-centered, but are not best-response compatible. For instance, it is easy to see that every sequence $\left(\alpha_{k}\right)_{k \in K^{-}}$such that for every $k \in K^{-}, \alpha_{k} \in[0, \min \{\alpha / 2,1 / 2\}]$, is both $\alpha$-centered and better-response compatible.

The restriction of being $\alpha$-centered on a better-response dynamic captures a form of cautiousness by the players, as they do not engage in updates that take them very far from their optimal choice. For finite values of $\alpha$, players do not change their action in an $\alpha$-centered path if they are at their best response, as that would imply $\left|\alpha_{k}\right|=\infty$. Alternatively, $\alpha$ can be thought of as a measure of inaccuracy of best-responding players who are unable to execute their intended strategies.

For the remainder of this paper we mainly consider $\alpha$-centered paths with $\alpha \in(0,1)$. In these paths, every updating player moves closer to his current best response.

We define the overshooting coefficient $\widehat{\alpha}_{k}$ similar to $\alpha_{k}$, replacing the best-response function $b$ with the unconstrained best-response function $\widehat{b}$. For a path of action profiles $\left(x^{k}\right)_{k \in K}$, we define

$$
\widehat{\alpha}_{k}=\frac{x_{i^{k}}^{k+1}-\widehat{b}_{i^{k}}\left(x^{k}\right)}{x_{i^{k}}^{k}-\widehat{b}_{i^{k}}\left(x^{k}\right)}, \quad k \in K^{-} .
$$

In the following proposition we show the relation between the value of $\widehat{\alpha}_{k}$ and changes in the value of the potential as defined in Proposition 3.1. This relationship will prove crucial in our convergence analysis.

Proposition 4.5. Let a game $G \in \mathcal{G}$ be given.

1. Let $\left(x^{k}\right)_{k \in K}$ be a path of action profiles such that for every $k \in K^{-}, \widehat{\alpha}_{k} \in \mathbb{R}$. Then it holds that

$$
\phi\left(x^{k+1}\right)-\phi\left(x^{k}\right)=\frac{1}{2}\left(1-\widehat{\alpha}_{k}\right)\left(1+\widehat{\alpha}_{k}\right)\left(\widehat{b}_{i^{k}}\left(x^{k}\right)-x_{i^{k}}^{k}\right)^{2}, \quad k \in K^{-} .
$$

\section{The game has no 1-centered cycles.}

Proposition 4.5 says that in a path of action profiles, the change of the potential is only determined by the magnitude of $\widehat{\alpha}_{k}$. Each time the updating player gets closer to his unconstrained best response by his update, the value of the potential increases, and each time he gets further from the unconstrained best response, the value of the potential decreases. By this property we show that if every update moves the updating player closer to his best response, then better-response cycles cannot exist. This also implies the non-existence of best-response cycles. Notice that the cycle in Example 4.3 is not 1 -centered. Furthermore, notice that for $\alpha>1$, cycling is possible in an $\alpha$-centered path. For example, if we have $i^{1}=i^{2}$ and $\alpha_{1}=\alpha_{2}=-1$, then $\left(x^{1}, x^{2}, x^{3}\right)$ constitutes a cycle. This means that $\alpha \leq 1$ is a necessary and sufficient condition for the non-existence of $\alpha$-centered cycles.

\section{Convergence results}

This section presents the main results of the paper, the convergence of a general class of learning processes. Clearly, processes that are not 1-centered will not converge in general, although there are examples of processes and networks where this is possible, e.g. better-response dynamics in case of an empty network.

Intuition suggests that for 1-centered processes, where each player moves closer to his best response, convergence is guaranteed. However, a player may get farther away from his best response through the updates of the other players, meaning that subsequent updates for any given player are not necessarily smaller in magnitude than previous ones. As a first step to establish convergence, we show that the distance between consecutive elements of any $\alpha$-centered path with $\alpha<1$ converges to zero and that the distance between the current action and the best response to it approaches zero for an updating player.

Proposition 5.1. Let $\left(x^{k}\right)_{k \in \mathbb{N}}$ be an $\alpha$-centered path in a game $G \in \mathcal{G}$ such that $\alpha<1$. It holds that:

1. $\lim _{k \rightarrow \infty}\left\|x^{k+1}-x^{k}\right\|_{2}=0$.

2. $\lim _{k \rightarrow \infty}\left|b_{i^{k}}\left(x^{k}\right)-x_{i^{k}}^{k}\right|=0$. 
The first statement of Proposition 5.1 follows from the monotonicity and therefore the convergence of the values of the potential along an $\alpha$-centered path, by applying Proposition 4.5 to translate differences in the value of the potential to distances between action profiles. The second statement shows convergence to the best response for all updating players. In order to achieve convergence to a Nash equilibrium, we need convergence to the best response for all players. This can only be achieved if all players update regularly, otherwise nothing guarantees convergence for a player who, for instance, never updates. We therefore define the notion of updating in every $\ell$ periods, which is going to be the final condition for our main result.

Definition 5.2. Player $i \in I$ updates in every $\ell$ periods in a path of action profiles $\left(x^{k}\right)_{k \in \mathbb{N}}$ in a game $G \in \mathcal{G}$ if for every $k \in \mathbb{N}$ there exists $k^{\prime} \in\{k, \ldots, k+\ell-1\}$ such that either $\left[x_{i}^{k^{\prime}} \neq x_{i}^{k^{\prime}+1}\right]$ or $\left[x^{k^{\prime}}=x^{k^{\prime}+1}\right.$ and $\left.x_{i}^{k^{\prime}}=x_{i}^{k^{\prime}+1}=b_{i}\left(x^{k^{\prime}}\right)\right]$.

A player satisfies Definition 5.2 if in every length $\ell$ segment of the path there is an action profile at which he updated or there exists a pair of successive action profiles that are identical and the player is at his best response. This condition is quite general, e.g. if $\ell>n$, then it includes the possibility of fixing the order of updates across the players - provided that each player gets a turn.

We are ready to present our main results.

Theorem 5.3. Let $(\bar{x}, w, t) \in P$ and let $\left(x^{k}\right)_{k \in \mathbb{N}}$ be an $\alpha$-centered path in a game $G \in \mathcal{G}(\bar{x}, w, t)$. If $\alpha<1$ and every player updates in every $\ell$ periods for some $\ell \in \mathbb{N}$, then every cluster point of $\left(x^{k}\right)_{k \in \mathbb{N}}$ belongs to $\operatorname{NE}(\bar{x}, w, t)$. If, in addition, we have that $|\operatorname{NE}(\bar{x}, w, t)|<\infty$, then there exists $x^{*} \in \operatorname{NE}(\bar{x}, w, t)$ such that $\lim _{k \rightarrow \infty} x^{k}=x^{*}$.

Theorem 5.3 combines the results in Propositions 4.5 and 5.1. It identifies sufficient conditions for a large class of learning processes to converge. The conditions are as follows: first, every update must take the updating player closer to his current best response and, second, players must update regularly. Theorem 5.3 and Proposition 5.1 together imply that any such process will spend an arbitrarily long time in any $\varepsilon$-range of any cluster point of the process. The existence of such cluster points is guaranteed due to the compactness of the set of strategy profiles. Theorem 5.3 guarantees that each cluster point is a Nash equilibrium.

If the set of Nash equilibria is finite, then we get convergence to a single Nash equilibrium point. The latter condition holds generically as stated in Proposition 3.2. Since finiteness of the number of equilibria applies to all weighted networks provided that the vector of targets are generic, the theorem includes unweighted network games if targets are determined by some random process. Finally, it follows from the proof of the theorem that in case the process does not converge to a single Nash equilibrium, then the set of cluster points is an infinite connected set of Nash equilibria with the property that the potential function assigns equal value to them.

We conclude this section by an example illustrating the tightness of these sufficient conditions.

Example 5.4. Consider the case with no strategic interaction, $w=0$, and interior target values, for every $i \in I, 0<t_{i}<\bar{x}_{i}$. Then a game $G \in \mathcal{G}(\bar{x}, w, t)$ has a single Nash equilibrium, $x^{*}=t$. Since the Nash equilibrium set is finite, Theorem 5.3 applies. It is easy to see that to achieve convergence to the Nash equilibrium, the $\alpha<1$ condition cannot be weakened even in this simple case. Letting $\alpha=1$ allows for $\lim _{k \rightarrow \infty} \alpha_{k}=1$, which means that the conditions of Proposition 5.1 are no longer satisfied. In this case, the distance to the best reply, which is equal to the target value $t_{i}$, is no longer converging to zero. We have no convergence to the Nash equilibrium.

\section{Conclusion}

In this paper we consider weighted network games, a class of games with a very wide range of applications, where direct, pairwise player interactions are described by a matrix of interaction weights. We show that these games are best-response potential games and, generically, have a finite set of Nash equilibria.

Pairs of linked players can always benefit from jointly deviating in an interior equilibrium. Two players whose contributions are strategic substitutes of each other can jointly increase their actions to increase their payoffs, while players whose contributions are strategic complements can jointly decrease their actions to improve their payoffs. In case all players' actions are strategic substitutes or all players' actions are strategic complements, such deviations lead to Pareto improvements. Therefore, in general, equilibria are neither strong nor efficient.

We study a large class of better-response learning processes. The convergence properties of these processes are determined by their centering parameter, which indicates to what extent players can overshoot their best responses. If players move closer to the best response at each update, as is the case for best-response dynamics and better-response dynamics with a centering parameter of one, then the players get arbitrarily close to the set of Nash equilibria and converge to a single Nash equilibrium whenever the set of Nash equilibria is finite, which is generically the case. This is due to the fact that the best-response potential is symmetric around the best response of the players, hence moving closer to the best response increases the value of the potential. In the case of better-response dynamics with unrestricted overshooting, it is shown that cycles may arise.

The restrictions on overshooting that guarantee convergence to a Nash equilibrium in the general case are the same as in a trivial game with no strategic interaction. The reason for this is that the best-response potential can be shown to increase whenever an updating player moves closer to his best response, irrespective of the values of the interaction 
weights. Our results hence identify a rich class of learning processes that produce Nash equilibria, including cautious betterreply dynamics.

Topics that are left unexplored in this paper include asymmetric interaction weights. In this case the existence of a bestresponse potential is no longer guaranteed, and hence, best-response cycles may occur. Asymmetric interaction is a more general framework that allows the modeling of a wider range of decision making scenarios. Examples include pollution between two neighboring cities where one of the cities is upriver, hence it is affected far less by the pollution of its neighbor than vice versa. Another topic is the issue of inefficiency of equilibria. These inefficiencies may disappear in different possibly more centralized - classes of learning processes. Finally, the beliefs that shape the updates themselves are left unmodeled and unexplored in this paper. These topics are open for future research.

\section{Appendix A. Proofs}

Proof of Proposition 2.5

By differentiation of the payoff function we get

$$
\frac{\partial \pi_{i}\left(x_{i}, x_{-i}\right)}{\partial x_{i}}=f_{i}^{\prime}\left(\sum_{j \in I} w_{i j} x_{j}\right)-c_{i} .
$$

The first order condition of unconstrained maximization is satisfied if $f_{i}^{\prime}\left(\sum_{j \in I} w_{i j} x_{j}\right)-c_{i}=0$. Using property (3) of $f_{i}$ this is satisfied if $\sum_{j \in I} w_{i j} x_{j}=t_{i}$.

If $t_{i}-\sum_{j \neq i} w_{i j} x_{j} \in\left[0, \bar{x}_{i}\right]$, then it follows that $b_{i}(x)=t_{i}-\sum_{j \neq i} w_{i j} x_{j}$. Note that the second order condition of maximization is satisfied due to the concavity of $f_{i}$, and, therefore, of $\pi_{i}$.

If $t_{i}-\sum_{j \neq i} w_{i j} x_{j}<0$, then for every $x_{i} \in X_{i}$ it holds that $t_{i}<\sum_{j \in I} w_{i j} x_{j}$. Invoking properties (2) and (3) of $f_{i}$, for every $x_{i} \in X_{i}$ we have $f_{i}^{\prime}\left(\sum_{j \in I} w_{i j} x_{j}\right)<c_{i}$, meaning that $\partial \pi_{i}\left(x_{i}, x_{-i}\right) / \partial x_{i}$ is uniformly negative. Therefore, $\pi_{i}$ is maximized for the lowest possible value of $x_{i}$, so $b_{i}(x)=0$.

Similarly, if $t_{i}-\sum_{j \neq i} w_{i j} x_{j}>\bar{x}_{i}$, then for every $x_{i} \in X_{i}$ it holds that $t_{i}>\sum_{j \in I} w_{i j} x_{j}$. Properties (2) and (3) of $f_{i}$ guarantee that for every $x_{i} \in X_{i}$ we have $f_{i}^{\prime}\left(\sum_{j \in I} w_{i j} x_{j}\right)>c_{i}$, and that $\partial \pi_{i}\left(x_{i}, x_{-i}\right) / \partial x_{i}$ is uniformly positive. Therefore, $\pi_{i}$ is maximized for the highest possible value of $x_{i}$, so $b_{i}(x)=\bar{x}_{i}$. This concludes the proof.

\section{Proof of Proposition 3.1}

We show that for every $x \in X$ and every $i \in I$ it holds that

$$
\underset{x_{i} \in X_{i}}{\operatorname{argmax}} \pi_{i}\left(x_{i}, x_{-i}\right)=\underset{x_{i} \in X_{i}}{\operatorname{argmax}} \phi\left(x_{i}, x_{-i}\right) .
$$

1. The left-hand side of the equality above equals $b_{i}(x)$. For the right-hand side, notice that $x^{\top} W$ is the row vector of consumption levels of each player, $x^{\top} W=\left(\sum_{j \in I} w_{1 j} x_{j}, \ldots, \sum_{j \in I} w_{n j} x_{j}\right)$, using the fact that $W$ is symmetric. Multiplication by $x$ gives

$$
x^{\top} W x=x_{1} \sum_{j \in I} w_{1 j} x_{j}+\cdots+x_{n} \sum_{j \in I} w_{n j} x_{j} .
$$

Differentiating $\phi\left(x_{i}, x_{-i}\right)$ by $x_{i}$ leads to

$$
\frac{\partial \phi\left(x_{i}, x_{-i}\right)}{\partial x_{i}}=t_{i}-\frac{1}{2}\left(2 x_{i}+\sum_{j \neq i} w_{i j} x_{j}+\sum_{j \neq i} w_{j i} x_{j}\right)=t_{i}-\sum_{i \in I} w_{i j} x_{j},
$$

where the last equality uses the symmetry of $W$.

Setting the derivative of $\phi$ with respect to $x_{i}$ equal to zero gives the extreme point $x_{i}=t_{i}-\sum_{j \neq i} w_{i j} x_{j}$, and as long as $t_{i}-\sum_{j \neq i} w_{i j} x_{j} \in\left[0, \bar{x}_{i}\right]$, it is the unique maximum, since the second derivative is $-w_{i i}=-1$.

If $t_{i}-\sum_{j \neq i} w_{i j} x_{j}<0$, then, since $x_{i} \geq 0$, the first derivative of $\phi$ with respect to $x_{i}$ is uniformly negative on $\left[0, \bar{x}_{i}\right]$ hence the unique maximum is achieved for the minimal contribution, $x_{i}=0$.

Similarly, if $t_{i}-\sum_{j \neq i} w_{i j} x_{j}>\bar{x}_{i}$, then the first derivative is uniformly positive, meaning that, in $\left[0, \bar{x}_{i}\right]$, the unique maximum is achieved for the maximal contribution, $x_{i}=\bar{x}_{i}$.

2. Let $b: X \rightarrow X$ be the function such that its component $i \in I$ is equal to $b_{i}$, the best-response function of player $i$. Since $X$ is non-empty, compact, and convex, and $b$ is continuous, the existence of an $x^{*}$ such that $b\left(x^{*}\right)=x^{*}$ is guaranteed by Brouwer's fixed-point theorem.

3. Since every game in $\mathcal{G}(\bar{x}, w, t)$ is a best-response potential game with potential $\phi$, every Nash equilibrium satisfies the stated KKT conditions.

Since, for every $i \in I$, for every $x \in X$, it holds that

$$
\frac{\partial^{2} \phi(x)}{\partial x_{i}^{2}}=-1<0,
$$

every point satisfying the KKT conditions yields a Nash equilibrium. 
Proof of Proposition 3.2

We use the characterization of Proposition 3.1 to derive conditions for the interaction matrix and the vector of targets that guarantee the equilibrium set to be finite. For $(\bar{x}, w, t) \in P$, let $\operatorname{KKT}(\bar{x}, w, t)$ denote the set of solutions $(x, \lambda, \mu)$ to the KKT conditions of Proposition 3.1. For $H \subseteq I$, let

$$
\operatorname{KKT}_{H}(\bar{x}, w, t)=\left\{(x, \lambda, \mu) \in \operatorname{KKT}(\bar{x}, w, t): \forall i \in H, \lambda_{i}=\mu_{i}=0 \text {, and } \forall i \in I \backslash H, \max \left\{\lambda_{i}, \mu_{i}\right\}>0\right\} .
$$

In words, $\operatorname{KKT}_{H}(\bar{x}, w, t)$ denotes the set of solutions to the KKT conditions of Proposition 3.1 such that players outside $H$ are either contributing zero or contributing up to their capacity. Of course, both cannot hold simultaneously and for each of these players one of the multipliers must equal zero.

The set $\mathrm{NE}_{H}(\bar{x}, w, t)$ is obtained by taking the projection of $\operatorname{KKT}_{H}(\bar{x}, w, t)$ to the set of action profiles $X, \mathrm{NE}_{H}(\bar{x}, w, t)=$ $\operatorname{proj}_{X} \operatorname{KKT}_{H}(\bar{x}, w, t)$, where $\operatorname{proj}_{X}$ is the projection mapping into $X$. If $x^{*} \in \operatorname{NE}_{H}(\bar{x}, w, t)$, then $x^{*} \in \mathrm{NE}(\bar{x}, w, t)$ and for every $i \in I \backslash H$ we have $x_{i}^{*} \in\left\{0, \bar{x}_{i}\right\}$. It follows that $\mathrm{NE}(\bar{x}, w, t)=\bigcup_{H \subset I} \mathrm{NE}_{H}(\bar{x}, w, t)$. The set of Nash equilibria $\mathrm{NE}(\bar{x}, w, t)$ is finite if and only if for every $H \subseteq I, \mathrm{NE}_{H}(\bar{x}, w, t)$ is finite.

Let bounds $\bar{x} \in \mathbb{R}_{++}^{n}$, interaction weights $w \in \mathbb{R}^{n(n-1) / 2}$, and a set $H \subseteq I$ be given. The set of target vectors for which $\mathrm{NE}_{H}(\bar{x}, w, t)$ is infinite is denoted by

$$
T_{H}=\left\{t \in \mathbb{R}^{n}:\left|\mathrm{NE}_{H}(\bar{x}, w, t)\right|=\infty\right\} .
$$

The set $\bar{T}_{H}$ denotes its closure. Further, let

$$
T=\left\{t \in \mathbb{R}^{n}:|\operatorname{NE}(\bar{x}, w, t)|=\infty\right\}
$$

denote the set of target vectors that yield infinitely many Nash equilibria and let $\bar{T}$ denote the closure of $T$.

Lemma A.1. For every $\bar{x} \in \mathbb{R}_{++}^{n}$, for every $w \in \mathbb{R}^{n(n-1) / 2}$, for every $H \subseteq I$, the set $\bar{T}_{H}$ has Lebesgue measure zero.

Proof. Let some $\bar{x} \in \mathbb{R}_{++}^{n}$, some $w \in \mathbb{R}^{n(n-1) / 2}$, and some $H \subseteq I$ be given.

First consider the case $H=\emptyset$. Then, for every $t \in \mathbb{R}^{n}$ it holds that $\mathrm{NE}_{\emptyset}(\bar{x}, w, t) \subseteq \prod_{i \in I}\left\{0, \bar{x}_{i}\right\}$, meaning that for every $t \in \mathbb{R}^{n}$ we have $\left|\mathrm{NE}_{\emptyset}(\bar{x}, w, t)\right|<\infty$. It follows that $T_{\emptyset}=\bar{T}_{\emptyset}=\emptyset$.

Now consider the case $H \neq \emptyset$. We show that there exists a set $U_{H} \subset \mathbb{R}^{n}$ of Lebesgue measure zero such that $\bar{T}_{H} \subseteq U_{H}$.

For every $t \in \mathbb{R}^{n}$, for every $x^{*} \in \mathrm{NE}_{H}(\bar{x}, w, t)$, we have that

$$
\begin{array}{ll}
x_{i}^{*} \in\left\{0, \bar{x}_{i}\right\}, & i \in I \backslash H, \\
t_{i}-\sum_{j \in H} w_{i j} x_{j}^{*}-\sum_{j \in I \backslash H} w_{i j} x_{j}^{*}=0, & i \in H .
\end{array}
$$

Let $W_{H}=\left(w_{i j}\right)_{i, j \in H}$ denote the submatrix of $W$ that we obtain by removing every row and every column whose index is not contained in $H$. Further, let $W_{H,-H}=\left(w_{i j}\right)_{i \in H, j \in I \backslash H}, t_{H}=\left(t_{i}\right)_{i \in H}, x_{H}^{*}=\left(x_{i}^{*}\right)_{i \in H}$, and $x_{-H}^{*}=\left(x_{i}^{*}\right)_{i \in I \backslash H}$. Now, the previous system of equations can be written in matrix form as

$$
W_{H} x_{H}^{*}=t_{H}-W_{H,-H} x_{-H}^{*}
$$

Therefore, by the Rouché-Capelli theorem, $\left|\mathrm{NE}_{H}(\bar{x}, w, t)\right|=\infty \operatorname{implies} \operatorname{rank}\left(W_{H}\right)<|H|$. So $T_{H}=\bar{T}_{H}=\emptyset$ whenever $\operatorname{rank}\left(W_{H}\right)=$ $|H|$. Consider the case where $\operatorname{rank}\left(W_{H}\right)<|H|$. For $y \in \prod_{i \in I \backslash H}\left\{0, \bar{x}_{i}\right\}$, let $U_{H}^{y}$ be the set of target vectors $t$ such that $t_{H}-W_{H,-H} y$ belongs to the span of $W_{H}$. Notice that $U_{H}^{y}$ is an $\left(n-|H|+\operatorname{rank}\left(W_{H}\right)\right)$-dimensional vector space and therefore a closed set of Lebesgue measure zero. Let $U_{H}=\bigcup_{y \in \prod_{i \in I}\left\{0, \bar{x}_{i}\right\}} U_{H}^{y}$. Since $U_{H}$ is a union of finitely many closed sets of Lebesgue measure zero, it is also closed and is of Lebesgue measure zero. Notice that $t \in \mathbb{R}^{n} \backslash U_{H}$ implies that $t \in \mathbb{R}^{n} \backslash T_{H}$, since for every $t \in \mathbb{R}^{n} \backslash U_{H}$ the system

$$
W_{H} x_{H}^{*}=t_{H}-W_{H,-H} x_{-H}^{*}
$$

has no solutions in $x_{H}^{*}$. It follows that $T_{H} \subseteq U_{H}$. Furthermore, since $U_{H}$ is closed, we also have $\bar{T}_{H} \subseteq U_{H}$.

We prove Lemma A.1 for the closure of the set $T_{H}$, which implies that the set of target vectors with infinitely many Nash equilibria is not only small in a measure theoretic sense, but also in a topological sense.

The intuition behind Lemma A.1 is that for a fixed subset of players, the set of interior Nash equilibria corresponds to the solution set of a linear system which, generically, has only one solution. The case of infinitely many solutions, and hence, the possibility of infinitely many Nash equilibria obtains only if the rank of the interaction matrix is not full and the target vector belongs to a vector space parallel to the span of the interaction matrix, which is only the case for a set of target vectors of Lebesgue measure zero.

As an illustation of Lemma A.1 for the case where all targets are very large or very small, i.e. for every $i \in I$ we have $t_{i}>\max _{x \in X} \sum_{j \in I} w_{i j} x_{j}$ or $t_{i}<\min _{x \in X} \sum_{j \in I} w_{i j} x_{j}$, the set of Nash equilibria is a subset of the corners of the strategy space $X$, i.e. $\operatorname{NE}(\bar{x}, w, t) \subseteq \prod_{i \in I}\left\{0, \bar{x}_{i}\right\}$, and is therefore finite.

Lemma A.2. For every $\bar{x} \in \mathbb{R}_{++}^{n}$, for every $w \in \mathbb{R}^{n(n-1) / 2}$, the set $\bar{T}$ has Lebesgue measure zero.

Proof. We show that there exists a set $U \subseteq \mathbb{R}^{n}$ of Lebesgue measure zero such that $\bar{T} \subseteq U$. 
Let $U=\bigcup_{H \subseteq I} U_{H}$. Since $U$ is a union of finitely many sets of Lebesgue measure zero, it has Lebesgue measure zero. Since $T=\bigcup_{H \subseteq I} T_{H}$, and $T_{H} \subseteq U_{H}$ for every $H \subseteq I$, it also holds that $T \subseteq U$. Once again, since $U$ is closed, we have $\bar{T} \subseteq U$.

Lemma A.2 shows that for any vector of bounds and any interaction structure the set of target vectors that induce infinitely many Nash equilibria is of null measure. Furthermore, since the closure of this set is also of null measure, the set of Nash equilibria is finite for almost every target vector, and the infinite cases are not dense in any positive measure subset of $\mathbb{R}^{n}$.

\section{Proof of Proposition 3.4}

1. We first discuss the case $w_{i j}>0$.

Since $x_{i}^{*} \in\left(0, \bar{x}_{i}\right)$ and $x_{j}^{*} \in\left(0, \bar{x}_{j}\right)$, expression (1) in Proposition 2.5 implies that $\sum_{k \in I} w_{i k} x_{k}=t_{i}$ and $\sum_{k \in I} w_{j k} x_{k}=t_{j}$. Hence, for $\delta>0$ such that $x_{i}^{*}+\delta \leq \bar{x}_{i}$ and $x_{j}^{*}+\delta \leq \bar{x}_{j}$, we have

$$
\frac{\pi_{i}\left(x^{*}+\delta^{\{i, j\}}\right)-\pi_{i}\left(x^{*}\right)}{\delta}=\frac{f_{i}\left(t_{i}+\delta\left(1+w_{i j}\right)\right)-f_{i}\left(t_{i}\right)}{\delta}-c_{i} .
$$

Since $f_{i}$ is concave, we have

$$
f_{i}\left(t_{i}+\delta\right) \leq f_{i}\left(t_{i}+\delta\left(1+w_{i j}\right)\right)-\delta w_{i j} f_{i}^{\prime}\left(t_{i}+\delta\left(1+w_{i j}\right)\right)
$$

Therefore, we can write

$$
\frac{\pi_{i}\left(x^{*}+\delta^{\{i, j\}}\right)-\pi_{i}\left(x^{*}\right)}{\delta} \geq \frac{f_{i}\left(t_{i}+\delta\right)-f_{i}\left(t_{i}\right)}{\delta}-c_{i}+w_{i j} f_{i}^{\prime}\left(t_{i}+\delta\left(1+w_{i j}\right)\right) .
$$

Let $\varepsilon_{i}=\min _{x \in X} f_{i}^{\prime}\left(\sum_{k \in I} w_{i k} x^{k}\right)$. Since $f_{i}^{\prime}$ is a continuous function, its minimum over the compact set $X$ is well-defined. Notice that Assumption 2.2 guarantees that $\varepsilon_{i}>0$. Thus,

$$
\frac{\pi_{i}\left(x^{*}+\delta^{\{i, j\}}\right)-\pi_{i}\left(x^{*}\right)}{\delta} \geq \frac{f_{i}\left(t_{i}+\delta\right)-f_{i}\left(t_{i}\right)}{\delta}-c_{i}+w_{i j} \varepsilon_{i} .
$$

Also due to the continuity of $f_{i}^{\prime}$, the term $\left(f_{i}\left(t_{i}+\delta\right)-f_{i}\left(t_{i}\right)\right) / \delta-c_{i}$ converges to zero as $\delta$ goes to zero. Hence, for sufficiently small positive $\delta$, we have $\pi_{i}\left(x^{*}+\delta^{\{i, j\}}\right)-\pi_{i}\left(x^{*}\right)>0$. The same argument applies to agent $j$.

The case $w_{i j}<0$ follows from very similar arguments.

2. We first consider the case where $w_{i j}>0$. As per Proposition 3.4 , there exists $\delta \in\left(0, \min \left\{x_{i}^{*}, x_{j}^{*}, \bar{x}_{i}-x_{i}^{*}, \bar{x}_{j}-x_{j}^{*}\right\}\right)$ such that $\pi_{i}\left(x^{*}+\delta^{\{i, j\}}\right)>\pi_{i}\left(x^{*}\right)$ and $\pi_{j}\left(x^{*}+\delta^{\{i, j\}}\right)>\pi_{j}\left(x^{*}\right)$. Since $w \geq 0$ it follows that for every other player $h \in I \backslash\{i, j\}$ we have $f_{h}\left(\sum_{k \in I} w_{h k} x_{k}^{*}+\delta^{\{i, j\}}\right) \geq f_{h}\left(\sum_{k \in I} w_{h k} x_{k}^{*}\right)$, while his own action did not change, and therefore $\pi_{h}\left(x^{*}+\delta^{\{i, j\}}\right) \geq \pi_{h}\left(x^{*}\right)$, meaning that players $i$ and $j$ increasing their action by $\delta$ yields a Pareto improvement.

The case $w \leq 0$ follows from similar arguments.

\section{Proof of Proposition 4.5}

The relationship between $\alpha_{k}$ and $\widehat{\alpha}_{k}$ is summarized in the following lemma.

Lemma A.3. Let $\left(x^{k}\right)_{k \in K}$ be a path of action profiles in a game $G \in \mathcal{G}$. The following statements hold for every $k \in K^{-}$:

(i) $\alpha_{k} \neq \widehat{\alpha}_{k}$ implies $b_{i^{k}}\left(x^{k}\right) \in\left\{0, \bar{x}_{i k}\right\}$.

(ii) $\alpha_{k} \in(0,1)$ implies $\widehat{\alpha}_{k} \in(0,1)$.

(iii) $\alpha_{k} \in(-1,0)$ implies $\alpha_{k}=\widehat{\alpha}_{k}$.

(iv) $\alpha_{k}=0$ implies $0 \leq \widehat{\alpha}_{k} \leq 1$.

(v) $\widehat{\alpha}_{k}=1$ implies $x^{k+1}=x^{k}$.

Proof. (i). If $b_{i^{k}}\left(x^{k}\right) \in\left(0, \bar{x}_{i^{k}}\right)$, then it holds that $b_{i^{k}}\left(x^{k}\right)=\widehat{b}_{i^{k}}\left(x^{k}\right)$ and thus $\alpha_{k}=\widehat{\alpha}_{k}$.

(ii). We only need to consider the case $\alpha_{k} \neq \widehat{\alpha}_{k}$. By (i) we have $b_{i^{k}}\left(x^{k}\right) \in\left\{0, \bar{x}_{i^{k}}\right\}$. Take the case $b_{i^{k}}\left(x^{k}\right)=0$. Then it holds that $\widehat{b}_{i^{k}}\left(x^{k}\right)<0$, so $x_{i^{k}}^{k+1}<x_{i^{k}}^{k}$ due to $0<\alpha_{k}<1$, and thus

$$
0<\alpha_{k}=\frac{x_{i^{k}}^{k+1}}{x_{i^{k}}^{k}}<\frac{x_{i^{k}}^{k+1}-\widehat{b}_{i^{k}}\left(x^{k}\right)}{x_{i^{k}}^{k}-\widehat{b}_{i^{k}}\left(x^{k}\right)}=\widehat{\alpha}_{k}<1 .
$$

The case $b_{i^{k}}\left(x^{k}\right)=\bar{x}_{i^{k}}$ follows from similar arguments.

(iii). Since $\alpha_{k} \in(-1,0)$, we have

$$
\operatorname{sgn}\left(x_{i^{k}}^{k}-b_{i^{k}}\left(x^{k}\right)\right)=-\operatorname{sgn}\left(x_{i^{k}}^{k+1}-b_{i^{k}}\left(x^{k}\right)\right) \neq 0 .
$$

Therefore, it must hold that $b_{i^{k}}\left(x^{k}\right) \in\left(0, \bar{x}_{i}\right)$, otherwise $x_{i^{k}}^{k+1}$ would not be in $X_{i^{k}}$. It follows that $b_{i^{k}}\left(x^{k}\right)=\widehat{b}_{i^{k}}\left(x^{k}\right)$. 
(iv). Once again, we only need to discuss the case $\alpha_{k} \neq \widehat{\alpha}_{k}$, so $b_{i^{k}}\left(x^{k}\right) \in\left\{0, \bar{x}_{i^{k}}\right\}$. Consider the case $b_{i^{k}}\left(x^{k}\right)=0$. We have that $\widehat{b}_{i^{k}}\left(x^{k}\right)<0$ and $x_{i^{k}}^{k+1}=0$ since $\alpha_{k}=0$. It holds that

$$
0=\alpha_{k} \leq \frac{-\widehat{b}_{i^{k}}\left(x^{k}\right)}{x_{i^{k}}^{k}-\widehat{b}_{i^{k}}\left(x^{k}\right)}=\widehat{\alpha}_{k} \leq 1 .
$$

The case $b_{i k}\left(x^{k}\right)=\bar{x}_{i k}$ follows from similar arguments.

(v). In case $\widehat{\alpha}_{k}=1$, we have

$$
x_{i^{k}}^{k+1}-\widehat{b}_{i^{k}}\left(x^{k}\right)=x_{i^{k}}^{k}-\widehat{b}_{i^{k}}\left(x^{k}\right),
$$

so $x_{i^{k}}^{k+1}=x_{i^{k}}^{k}$.

1. Using the definition of $\phi$ gives

$$
\phi\left(x^{k+1}\right)-\phi\left(x^{k}\right)=\sum_{i \in I}\left(x_{i}^{k+1}-x_{i}^{k}\right) t_{i}-\frac{1}{2} \sum_{i \in I} x_{i}^{k+1}\left(\sum_{j \in I} w_{i j} x_{j}^{k+1}\right)+\frac{1}{2} \sum_{i \in I} x_{i}^{k}\left(\sum_{j \in I} w_{i j} x_{j}^{k}\right) .
$$

Using the symmetry of the interaction matrix $W$ and taking advantage of the fact that $x^{k+1}$ is the successor of $x^{k}$ in a path, we substitute $x_{-i^{k}}^{k}=x_{-i^{k}}^{k+1}$ to get

$$
\phi\left(x^{k+1}\right)-\phi\left(x^{k}\right)=\left(x_{i^{k}}^{k+1}-x_{i^{k}}^{k}\right) t_{i^{k}}-\frac{1}{2}\left(\left(x_{i^{k}}^{k+1}\right)^{2}-\left(x_{i^{k}}^{k}\right)^{2}\right)-\left(x_{i^{k}}^{k+1}-x_{i^{k}}^{k}\right) \sum_{j \neq i^{k}} w_{i^{k}} x_{j}^{k} .
$$

Factoring out $x_{i^{k}}^{k+1}-x_{i^{k}}^{k}$ yields

$$
\phi\left(x^{k+1}\right)-\phi\left(x^{k}\right)=\left(x_{i^{k}}^{k+1}-x_{i k}^{k}\right)\left[\left(t_{i^{k}}-\frac{1}{2}\left(x_{i^{k}}^{k+1}+x_{i^{k}}^{k}\right)-\sum_{j \neq i^{k}} w_{i^{k} j} x_{j}^{k}\right] .\right.
$$

Substituting $\widehat{b}_{i^{k}}\left(x^{k}\right)=t_{i^{k}}-\sum_{j \neq i^{k}} w_{i^{k} j} x_{j}^{k}$ gives

$$
\begin{aligned}
\phi\left(x^{k+1}\right)-\phi\left(x^{k}\right) & =\left(x_{i^{k}}^{k+1}-x_{i^{k}}^{k}\right)\left(\hat{b}_{i^{k}}\left(x^{k}\right)-\frac{1}{2}\left(x_{i^{k}}^{k+1}+x_{i^{k}}^{k}\right)\right) \\
& =\frac{1}{2}\left(x_{i^{k}}^{k+1}-x_{i^{k}}^{k}\right)\left[\widehat{b}_{i^{k}}\left(x^{k}\right)-x_{i^{k}}^{k+1}+\widehat{b}_{i^{k}}\left(x^{k}\right)-x_{i^{k}}^{k}\right] .
\end{aligned}
$$

Finally, substituting $x_{i^{k}}^{k+1}=\left(1-\widehat{\alpha}_{k}\right) \widehat{b}_{i^{k}}\left(x^{k}\right)+\widehat{\alpha}_{k^{\prime}} x_{i^{k}}^{k}$ and $\widehat{b}_{i^{k}}\left(x^{k}\right)-x_{i^{k}}^{k+1}=\widehat{\alpha}_{k}\left(\widehat{b}_{i^{k}}\left(x^{k}\right)-x_{i^{k}}^{k}\right)$ gives

$$
\phi\left(x^{k+1}\right)-\phi\left(x^{k}\right)=\frac{1}{2}\left(1-\widehat{\alpha}_{k}\right)\left(1+\widehat{\alpha}_{k}\right)\left(\widehat{b}_{i^{k}}\left(x^{k}\right)-x_{i^{k}}^{k}\right)^{2} .
$$

2. Suppose that $\left(x^{k}\right)_{k \in K}$ is a 1 -centered cycle. For every $k \in K^{-}$it holds by Lemma A.3 that $\widehat{\alpha}_{k} \in(-1,1]$ and therefore by the first statement we have $\phi\left(x^{k+1}\right)-\phi\left(x^{k}\right) \geq 0$.

By Definition 4.1, each path has at least one pair of successive action profiles that are different. Let $k^{\prime} \in K^{-}$be such that $x^{k^{\prime}+1} \neq x^{k^{\prime}}$. Since the path $\left(x^{k}\right)_{k \in K}$ is 1 -centered, Lemma A.3 implies $\left|\widehat{\alpha}_{k^{\prime}}\right|<1$, and therefore by the first statement we have $\phi\left(x^{k^{\prime}+1}\right)-\phi\left(x^{k^{\prime}}\right)>0$. Together with the fact that $\phi\left(x^{k+1}\right)-\phi\left(x^{k}\right) \geq 0$ for every $k \in K^{-}$, we obtain a contradiction to $\left(x^{k}\right)_{k \in K}$ being a 1-centered cycle.

\section{Proof of Proposition 5.1}

1. We use the fact that $x_{i^{k}}^{k+1}=\left(1-\widehat{\alpha}_{k}\right) \widehat{b}_{i^{k}}\left(x^{k}\right)+\widehat{\alpha}_{k^{k}} x_{i k}^{k}$ to obtain

$$
\left\|x^{k+1}-x^{k}\right\|_{2}^{2}=\left(x_{i^{k}}^{k+1}-x_{i^{k}}^{k}\right)^{2}=\left(1-\widehat{\alpha}_{k}\right)^{2}\left(\widehat{b}_{i^{k}}\left(x^{k}\right)-x_{i^{k}}^{k}\right)^{2} .
$$

Applying Proposition 4.5 gives

$$
\left\|x^{k+1}-x^{k}\right\|_{2}^{2}=2 \frac{1-\widehat{\alpha}_{k}}{1+\widehat{\alpha}_{k}}\left(\phi\left(x^{k+1}\right)-\phi\left(x^{k}\right)\right) .
$$

Since the path is $\alpha$-centered with $\alpha<1$, by parts (ii)-(iv) of Lemma A.3 we have $-\alpha<\widehat{\alpha}_{k} \leq 1$. It follows that

$$
\left\|x^{k+1}-x^{k}\right\|_{2}^{2} \leq 2 \frac{1+\alpha}{1-\alpha}\left(\phi\left(x^{k+1}\right)-\phi\left(x^{k}\right)\right) .
$$

By the first part of Proposition 4.5 we have that the sequence $\left(\phi\left(x^{k}\right)\right)_{k \in \mathbb{N}}$ is monotonically increasing. Furthermore, since $\phi$ is continuous and the set $X$ is compact, the sequence $\left(\phi\left(x^{k}\right)\right)_{k \in \mathbb{N}}$ is also bounded, and hence it is convergent, so $\phi\left(x^{k+1}\right)-$ $\phi\left(x^{k}\right) \rightarrow 0$ as $k \rightarrow \infty$. Since the right-hand side of (4) converges to zero, it follows that $\left\|x^{k+1}-x^{k}\right\|_{2}^{2} \rightarrow 0$ as $k \rightarrow \infty$. This implies the statement.

2. Suppose it does not hold that $\lim _{k \rightarrow \infty}\left|b_{i^{k}}\left(x^{k}\right)-x_{i k}^{k}\right|=0$. Then the sequence $\left(i^{k}, x^{k}\right)_{k \in \mathbb{N}}$ has a converging subsequence $\left(i^{\ell}, x^{k^{\ell}}\right)$ with limit $(i, x)$ such that $\left|b_{i}(x)-x_{i}\right|=\varepsilon>0$. We distinguish three cases: (a) $\widehat{b}_{i}(x) \in\left(0, \bar{x}_{i}\right)$, (b) $\widehat{b}_{i}(x) \leq 0$, and (c) $\widehat{b}_{i}(x) \geq \bar{x}_{i}$.

Case (a). $\widehat{b}_{i}(x) \in\left(0, \bar{x}_{i}\right)$. 
There is $\ell^{\prime} \in \mathbb{N}$ such that, for every $\ell \geq \ell^{\prime}, i^{k^{\ell}}=i, b_{i}\left(x^{k^{\ell}}\right) \in\left(0, \bar{x}_{i}\right)$, and $\left|b_{i}\left(x^{k^{\ell}}\right)-x_{i}^{k^{\ell}}\right| \geq \varepsilon / 2$. From $\widehat{b}_{i}\left(x^{k^{\ell}}\right)=b_{i}\left(x^{k^{\ell}}\right)$ it follows that $\widehat{\alpha}_{k^{\ell}}=\alpha_{k^{\ell}}$, so by Proposition 4.5,

$$
\phi\left(x^{k^{\ell}+1}\right)-\phi\left(x^{k^{\ell}}\right) \geq \frac{1}{2}\left(1-\alpha_{k^{\ell}}\right)\left(1+\alpha_{k^{\ell}}\right) \frac{1}{4} \varepsilon^{2}>\frac{1}{2}(1-\alpha)(1+\alpha) \frac{1}{4} \varepsilon^{2}, \quad \ell \geq \ell^{\prime} .
$$

By Proposition 4.5, we have that the sequence $\left(\phi\left(x^{k}\right)\right)_{k \in \mathbb{N}}$ is monotonically increasing, so the subsequence $\left(\phi\left(x^{k^{\ell}}\right)\right)_{\ell \in \mathbb{N}}$ is monotonically increasing, and by (5) it tends to infinity. This contradicts the fact that the continuous function $\phi$ has a maximum on the compact set $X$.

Case (b). $\widehat{b}_{i}(x) \leq 0$.

We have that $b_{i}(x)=0$ and $x_{i}=\varepsilon$. There is $\ell^{\prime} \in \mathbb{N}$ such that, for every $\ell \geq \ell^{\prime}, i^{k^{\ell}}=i, b_{i}\left(x^{k^{\ell}}\right) \leq x_{i}^{k^{\ell}}$, and $\left|b_{i}\left(x^{k^{\ell}}\right)-x_{i}^{k^{\ell}}\right| \geq \varepsilon / 2$. If $\widehat{b}_{i}\left(x^{k^{\ell}}\right) \geq 0$, then $\widehat{\alpha}_{k^{\ell}}=\alpha_{k^{\ell}}$. Otherwise, we have $\widehat{b}_{i}\left(x^{k^{\ell}}\right)<0$, so $b_{i}\left(x^{k^{\ell}}\right)=0$, and

$$
0 \leq \widehat{\alpha}_{k^{\ell}}=\frac{x_{i}^{k^{\ell}+1}-\widehat{b}_{i}\left(x^{k^{\ell}}\right)}{x_{i}^{k^{\ell}}-\widehat{b}_{i}\left(x^{k^{\ell}}\right)} \leq \frac{x_{i}^{k^{\ell}+1}-\underline{b}_{i}}{x_{i}^{k^{\ell}}-\underline{b}_{i}}=\frac{\alpha_{k^{\ell}} x_{i}^{k^{\ell}}-\underline{b}_{i}}{x_{i}^{k^{\ell}}-\underline{b}_{i}} \leq \frac{\frac{1}{2} \alpha_{k^{\ell}} \varepsilon-\underline{b}_{i}}{\frac{1}{2} \varepsilon-\underline{b}_{i}}<\frac{\frac{1}{2} \alpha \varepsilon-\underline{b}_{i}}{\frac{1}{2} \varepsilon-\underline{b}_{i}} .
$$

The right-hand side of $(6)$, denoted by $\beta$, belongs to $(\alpha, 1)$, so it holds that

$$
-\alpha<\widehat{\alpha}_{k^{\ell}} \leq \beta, \quad \ell \geq \ell^{\prime} .
$$

By Proposition 4.5, we have that

$$
\phi\left(x^{k^{\ell}+1}\right)-\phi\left(x^{k^{\ell}}\right) \geq \frac{1}{2}\left(1-\alpha_{k^{\ell}}\right)\left(1+\alpha_{k^{\ell}}\right) \frac{1}{4} \varepsilon^{2}>\frac{1}{2}(1-\beta)(1+\beta) \frac{1}{4} \varepsilon^{2}, \quad \ell \geq \ell^{\prime} .
$$

By Proposition 4.5, we have that the sequence $\left(\phi\left(x^{k}\right)\right)_{k \in \mathbb{N}}$ is monotonically increasing, so the subsequence $\left(\phi\left(x^{k^{\ell}}\right)\right)_{\ell \in \mathbb{N}}$ is monotonically increasing, and by (7) it tends to infinity. This contradicts the fact that the continuous function $\phi$ has a maximum on the compact set $X$.

Case $(\mathrm{c}) . \widehat{b}_{i}(x) \geq \bar{x}_{i}$.

We can derive a contradiction along similar lines as in Case (b).

Since all three cases lead to a contradiction, we conclude that $\lim _{k \rightarrow \infty}\left|b_{i^{k}}\left(x^{k}\right)-x_{i^{k}}^{k}\right|=0$.

\section{Proof of Theorem 5.3}

We first show the first statement. Since every linear function is Lipschitz continuous, the function $b_{i}: X \rightarrow X_{i}$ is Lipschitz continuous for every $i \in I$. Denote the Lipschitz constant of $b_{i}$ by $L_{i}$.

Let $x$ be a cluster point of $\left(x^{k}\right)_{k \in \mathbb{N}}$. We prove the result by showing that, for every $i \in I$, for every $\varepsilon>0,\left|b_{i}(x)-x_{i}\right|<\varepsilon$. We need a technical lemma. In this lemma we restrict attention to $\ell \geq 2$, which is implied by the second condition of a path in Definition 4.1 .

Lemma A.4. Let $\left(x^{k}\right)_{k \in \mathbb{N}}$ be a path of action profiles in a game $G \in \mathcal{G}$ such that $\lim _{k \rightarrow \infty}\left\|x^{k+1}-x^{k}\right\|_{2}=0$. For every $\varepsilon>0$, for every $\ell \in \mathbb{N}$ with $\ell \geq 2$, there exists $M \in \mathbb{N}$ such that, for every $m>M$, for every $k \in\{m, \ldots, m+\ell-1\}$, we have $\left\|x^{k}-x^{m}\right\|_{2}<\varepsilon$.

Proof. Let some $\varepsilon>0$ and some $\ell \in \mathbb{N}$ with $\ell \geq 2$ be given.

For every $\delta>0$ there exists $M_{\delta} \in \mathbb{N}$ such that for every $m>M_{\delta}$ we have $\left\|x^{m+1}-x^{m}\right\|_{2}<\delta$, since $\lim _{k \rightarrow \infty}\left\|x^{k+1}-x^{k}\right\|_{2}=0$. We take $\delta=\varepsilon /(\ell-1)$ and consider an arbitrary $m>M_{\delta}$.

Then, by the triangle inequality, for every $k \in\{m, \ldots, m+\ell-1\}$ we can write

$$
\left\|x^{k}-x^{m}\right\|_{2} \leq\left\|x^{k}-x^{k-1}\right\|_{2}+\cdots+\left\|x^{m+1}-x^{m}\right\|_{2}<\frac{k-m}{\ell-1} \varepsilon \leq \varepsilon .
$$

Therefore, $M_{\delta}$ is a suitable candidate for $M$.

Let $i \in I$ and $\varepsilon>0$ be given.

Let $M_{1} \in \mathbb{N}$ be such that, for every $m>M_{1}$, for every $k \in\{m, \ldots, m+\ell-1\}$, we have $\left\|x^{k}-x^{m}\right\|_{2}<\varepsilon /\left(3+2 L_{i}\right)$. Lemma A.4 guarantees the existence of such an $M_{1}$.

Let $M_{2} \in \mathbb{N}$ be such that for every $m>M_{2}$ it holds that $\left|b_{i m}\left(x^{m}\right)-x_{i m}^{m}\right|<\varepsilon /\left(3+2 L_{i}\right)$. The second statement of Proposition 5.1 guarantees the existence of such an $M_{2}$.

Let $m>\max \left\{M_{1}, M_{2}\right\}$ be such that $\left\|x^{m}-x\right\|_{2}<\varepsilon /\left(3+2 L_{i}\right)$. Such an $m$ must exist, since $x$ is a cluster point of the sequence $\left(x^{k}\right)_{k \in \mathbb{N}}$.

If player $i$ updates in every $\ell$ periods, then there exists $k^{\prime} \in\{m, \ldots, m+\ell-1\}$ such that $\left|b_{i}\left(x^{k^{\prime}}\right)-x_{i}^{k^{\prime}}\right|<\varepsilon /\left(3+2 L_{i}\right)$, where we use that $m>M_{2}$. Since $m>M_{1}$ as well, it holds that $\left\|x^{k^{\prime}}-x^{m}\right\|_{2}<\varepsilon /\left(3+2 L_{i}\right)$, and by the choice of $m$ we have $\left\|x-x^{m}\right\|_{2}<\varepsilon /\left(3+2 L_{i}\right)$. In particular, it follows that $\left|x_{i}^{k^{\prime}}-x_{i}^{m}\right|<\varepsilon /\left(3+2 L_{i}\right)$ and $\left|x_{i}^{m}-x_{i}\right|<\varepsilon /\left(3+2 L_{i}\right)$. By the triangle inequality we get

$$
\left|b_{i}\left(x^{k^{\prime}}\right)-x_{i}\right| \leq\left|b_{i}\left(x^{k^{\prime}}\right)-x_{i}^{k^{\prime}}\right|+\left|x_{i}^{k^{\prime}}-x_{i}^{m}\right|+\left|x_{i}^{m}-x_{i}\right|<\frac{3 \varepsilon}{3+2 L_{i}} .
$$

Also, $\left\|x-x^{m}\right\|_{2}<\varepsilon /\left(3+2 L_{i}\right)$ and $\left\|x^{m}-x^{k^{\prime}}\right\|_{2}<\varepsilon /\left(3+2 L_{i}\right)$ imply that $\left\|x-x^{k^{\prime}}\right\|_{2}<2 \varepsilon /\left(3+2 L_{i}\right)$. Using the Lipschitz continuity of $b_{i}$, we get

$$
\left|b_{i}(x)-b_{i}\left(x^{k^{\prime}}\right)\right|<\frac{2 L_{i} \varepsilon}{3+2 L_{i}} .
$$


Summing up, we have

$$
\left|b_{i}(x)-x_{i}\right| \leq\left|b_{i}(x)-b_{i}\left(x^{k^{\prime}}\right)\right|+\left|b_{i}\left(x^{k^{\prime}}\right)-x_{i}\right|<\frac{2 L_{i} \varepsilon}{3+2 L_{i}}+\frac{3 \varepsilon}{3+2 L_{i}}=\varepsilon .
$$

This concludes the first part of the proof.

For the second part let $Y$ denote the non-empty set of cluster points of $\left(x^{k}\right)_{k \in \mathbb{N}}$. The first statement implies that every element of $Y$ is a Nash equilibrium. We therefore only have to show that $Y$ is a singleton. We know that the set $Y$ is finite, since $Y \subseteq \mathrm{NE}(\bar{x}, w, t)$ and the set $\mathrm{NE}(\bar{x}, w, t)$ is finite by assumption.

Let some $y \in Y$ be given. Since the set $Y$ is finite, there exists $\varepsilon>0$ such that for every $x \in X \backslash\{y\}$ with $\|x-y\|_{2} \leq \varepsilon$ it holds that $\phi(x)-\phi(y)<0$. Take $\varepsilon>0$ sufficiently small such that the set

$$
D(y)=\left\{x \in X: \frac{\varepsilon}{2} \leq\|x-y\|_{2} \leq \varepsilon\right\}
$$

is non-empty. Since $D(y)$ is also compact, the number $\phi=\max _{x \in D(y)} \phi(x)$ is well-defined. Note that $\phi(y)>\phi$.

Since $\phi$ is continuous and $y \in Y$, we have that $\lim _{k \rightarrow \infty} \phi\left(x^{k}\right)=\phi(y)$. So there exists $M_{1} \in \mathbb{N}$ such that for every $k>M_{1}$ it holds that $\phi\left(x^{k}\right)>\phi$. Furthermore, since $\lim _{k \rightarrow \infty}\left\|x^{k+1}-x^{k}\right\|_{2}=0$ by Proposition 5.1, it holds that there exists $M_{2} \in \mathbb{N}$ such that for every $k>\bar{M}_{2}$ we have $\left\|x^{k+1}-x^{k}\right\|_{2}<\varepsilon / 2$.

Let $m>\max \left\{M_{1}, M_{2}\right\}$ be such that $\left\|x^{m}-y\right\|_{2}<\varepsilon / 2$. Such an $m$ must exist due to the fact that $y \in Y$. We argue that for every $k>m$ we have $\left\|x^{k}-y\right\|_{2}<\varepsilon / 2$. Suppose to the contrary that there exists $k>m$ with $\left\|x^{k}-y\right\|_{2} \geq \varepsilon / 2$ and let $k$ be the smallest such number. Since $k>M_{2}$ and $\left\|x^{k-1}-y\right\|_{2}<\varepsilon / 2$, we have $\left\|x^{k}-y\right\|_{2}<\varepsilon$, hence $x^{k} \in D(y)$ and $\phi\left(x^{k}\right) \leq \underline{\phi}<\phi\left(x^{m}\right)$, contradicting the fact that the sequence $\left(\phi\left(x^{k}\right)\right)_{k \in \mathbb{N}}$ is non-decreasing.

We have shown that for every $\varepsilon>0$ sufficiently small, there exists $m \in \mathbb{N}$ such that for every $k>m$ it holds that $\| x^{k}-$ $y \|_{2}<\varepsilon / 2$. It follows that $y$ is the only cluster point of $\left(x^{k}\right)_{k \in \mathbb{N}}$.

\section{Supplementary material}

Supplementary material associated with this article can be found, in the online version, at doi:10.1016/j.jedc.2019.06.004.

\section{References}

Allouch, N., 2015. On the private provision of public goods on networks. J. Econ. Theory 157, 527-552.

Ballester, C., Calvó-Armengol, A., 2010. Interactions with hidden complementarities. Reg. Sci. Urban Econ. 40, $397-406$.

Ballester, C., Calvó-Armengol, A., Zenou, Y., 2006. Who's who in networks. wanted: The key player. Econometrica 74, $1403-1417$.

Belhaj, M., Bramoullé, Y., Deroïan, F., 2014. Network games under strategic complementarities. Games Econ. Behav. 88, $310-319$.

Bervoets, S., Bravo, M., Faure, M., 2018. Learning with minimal information in continuous games arXiv:1806.11506v1.

Bervoets, S., Faure, M., 2019. Stability in games with continua of equilibria. J. Econ. Theory 179, 131-162.

Blume, L. E., Brock, W. A., Durlauf, S. N., Ioannides, Y. M., Identification of social interactions. In Handbook of Social Economics Benhabib, J., Bisin, A., Matthew, O. J., Amsterdam: North Holland, 853-964

Bourlès, R., Bramoullé, Y., Perez-Richet, E., 2017. Altruism in networks. Econometrica 85, 675-689.

Bramoullé, Y., Kranton, R., 2007. Public goods in networks. J. Econ. Theory 135, 478-494.

Bramoullé, Y., Kranton, R., D’Amours, M., 2014. Strategic interaction and networks. Am. Econ. Rev. 104, 898-930.

Cachon, G.P., Camerer, C., 1996. The sunk cost fallacy, forward induction and behavior in coordination games. Q. J. Econ. 111, $165-194$.

Cason, T., Friedman, D., 1997. Price formation in single call markets. Econometrica 65, 311-345.

Dubey, P., Haimanko, O., Zapechelnyuk, A., 2006. Strategic complements and substitutes, and potential games. Games Econ. Behav. 54, 77-94.

Eksin, C., Molavi, P., Ribeiro, A., Jadbabaie, A., 2012. Learning in linear games over networks. In: Communication, Control, and Computing (Allerton), 50th Annual Allerton Conference. IEEE, pp. 434-440.

Elliott, M., Golub, B., 2019. A network approach to public goods. J. Political Economy 127, 730-776.

Ewerhart, C., 2017. The lottery contest is a best-response potential game. Econ. Lett. 155, 168-171.

Fudenberg, D., Levine, D.K., 1998. The Theory of Learning in Games. MIT Press.

Goyal, S., 2012. Connections: An Introduction to the Economics of Networks. Princeton University Press.

Helsley, R.W., Zenou, Y., 2014. Social networks and interactions in cities. J. Econ. Theory 150, 426-466.

Jackson, M.O., Zenou, Y., 2014. Games on networks. In: Young, P., Zamir, S. (Eds.), Handbook of Game Theory, Vol. 4. Elsevier Science.

Jensen, M.K., 2010. Aggregative games and best-reply potentials. Econ. Theory 43, 45-66.

Kagel, J., Levin, D., 1999. Common value auctions with insider information. Econometrica 67, 1219-1238.

König, M., Liu, X., Zenou, Y., 2014. R\&d networks: Theory, empirics and policy implications. Forthcoming in. Rev. Econ. Stat.

Kukushkin, N.S., 2004. Best response dynamics in finite games with additive aggregation. Games Econ. Behav. 48, 94-110.

Kukushkin, N.S., 2015. Cournot tatonnement and potentials. J. Math. Econ. 59, 117-127.

Monderer, D., Shapley, L., 1996. Potential games. Games Econ. Behav. 14, 124-143.

Nagel, R., Vriend, N., 1999. An experimental study of adaptive behavior in an oligopolistic market game. J. Evol. Econ. 9, $27-65$.

Sandler, T., Hartley, K., 1995. The Economics of Defense. Cambridge University Press.

Sandler, T., Hartley, K., 2001. Economics of alliances: The lessons for collective action. J. Econ. Lit. 39, 869-896.

Sandler, T., Hartley, K., 2007. Handbook of Defense Economics: Defense in a globalized world. Elsevier.

Selten, R., Buchta, J., 1998. Experimental sealed bid first price auction with directly observed bid functions. In: Budescu, D., Erev, I., Zwick, R. (Eds.), Games and Human Behavior, Essays in Honor of Amnon Rapoport. Hillsdale, NJ.

Selten, R., Stoecker, R., 1986. End behavior in sequences of finite prisoner's dilemma supergames: A learning theory approach. J. Econ. Behav. Organ. 7, 47-70.

Voorneveld, M., 2000. Best-response potential games. Econ. Lett. 66, 289-295. 